\title{
Les économies alternatives dans les Corbières et la Haute Vallée de l'Aude: vers le travail non aliéné et l'approvisionnement communautaire
}

\author{
Rachel Slocum ${ }^{1}$ \\ Teresa Gowan \\ Graduate Institute of World Learning, USA \\ University of Minnesota, USA
}

\begin{abstract}
The Aude, a rural département in southwest France, is the site of one of the longest-lived European concentrations of counter-cultural practice. Here, villages abandoned by the devastating rural exodus post WWII were discovered by enthusiastic radicals of the '68 generation, who were able to establish themselves because property was cheap. From ethnographic research, we find that a receptive community, inexpensive resources and a strongly interventionist welfare state provide a fertile ground into which practitioners of plenitude may enact a non-normative approach to work and money, which takes root, grows and spreads. We identify a continuum of alternative economic practices encompassing a range of approaches to work itselfsome privileging a life of radical simplicity and autonomy, and others interested in developing more successful artisanal businesses. We find a place for neo-medievalists/ruralists who fetishize practices like the horse-drawn plough, and large-scale organic farmers. Finally, we show that the plenitude practices among the Aude alternatifs are tied together by communal reliance-by networks of support and cooperation that rely on being amongst and caring for others. Gifting and 'lending a hand' are key to these economies, enabling them to flourish. While this raises questions about the cost of living for those who do not participate for whatever reason in this exchange network, we see gifting as fundamental to long lasting alternative economies. This case refines our understanding of long-standing debates around the necessity and desirability of selfsufficiency.
\end{abstract}

Key words: alternative economies, communal reliance, sustainable agriculture, l'Aude, alienation

\section{Résumé}

L'Aude, un département rural du sud-ouest de la France, est le théâtre d'une des plus anciennes concentration de pratiques non-conformistes d'Europe. C'est ici que les villages ravagés par l'exode rural après la Seconde Guerre mondiale ont découvert les enthousiastes radicaux de la génération '68 qui ont pu s'y installer car les propriétés étaient bon marché. Dans le cadre de notre recherche ethnographique, nous constatons qu'une communauté réceptive, des ressources peu onéreuses et un État très interventionniste fournissent le sol fertile

\footnotetext{
${ }^{1}$ Les deux auteurs ont contribué également à cette publication. Dr. Rachel Slocum, Associate Professor, School for International Training, Graduate Institute of World Learning, 1 Kipling Road Brattleboro, VT 05301, USA. Email: rachel_slocum "at" hotmail.com. Dr. Teresa Gowan, Associate Professor, Department of Sociology, University of Minnesota, Minneapolis, MN 55455, USA. Email: tgowan "at" umn.edu. Cet article est une version révisée d'un chapitre (Gowan et Slocum 2014) publié dans la collection éditée par Juliet Schor et Craig Thompson (2014), Sustainable lifestyles and the quest for plenitude: case studies of the new economy. Dans cet article, nous nous concentrons plus sur l'exclusion sociale, de l'agriculture durable et de l'écologie politique. Nous publions cet article avec la permission de Yale University Press et le Journal of Political Ecology. Nos premiers remerciements vont aux petits producteurs qui ont gracieusement répondu à nos questions, prenant sur leur emploi du temps chargé et sur leur temps de loisir pour répondre à nos questions. L'argument présenté ici a été incité et amendé par des conversations avec Jack Atmore, Juliet Schor, Marianne Sica, Michel Taurignac, Ryan Galt, Valentine Cadieux, et les membres du groupe de lecture AgriFood à l'Université du Minnesota. En ce qui concerne l'assistance matérielle, nous sommes reconnaissantes envers Jack, Juliet, Tessa, le Département de Géographie et des Sciences de la Terre de l'Université du Wisconsin-La Crosse, et une bourse Imagine de l'Université du Minnesota. Nous remercions François-Nicolas Vozel pour sa traduction engagée et adroite, le conseil de l'éditeur de JPE, Simon Batterbury, et les recommandations des arbitres.
} 
sur lequel l'approche non-normative du travail et de l'argent des praticiens de plenitude ${ }^{2}$ peut prendre racine, se développer et se répandre. Nous identifions un continuum de pratiques économiques alternatives qui enveloppe un éventail d'approches du travail—certains privilégiant une vie de simplicité radicale et d'autonomie, et d'autres souhaitant développer des entreprises artisanales plus ambitieuses. Nous trouvons un lieu propice aux néo-médiévistes/ruraux qui fétichisent des pratiques telles que le labourage à l'aide de chevaux, et aux exploitations agricoles à grande échelle. Pour finir, nous montrons que les pratiques de la nouvelle richesse chez les alternatifs de l'Aude sont reliées entre elles par l'approvisionnement communautaire-par des réseaux de soutien et de coopération qui reposent sur le vivre-ensemble et le souci de l'autre. Le don et le 'coup de main' sont essentiels pour ces économies car ils leur permettent de prospérer. Même si cela soulève des questions au sujet du coût de la vie pour ceux qui ne participent pas à ce réseau d'échange pour une raison quelconque, nous voyons le don comme fondamental aux économies alternatives durables. Ce cas d'étude affine notre compréhension de débats de longue durée autour de la nécessité et de l'attrait de l'auto-sufficance.

Mots clés: économies alternatives, l'auto-approvisionnement, l'agriculture bio, véritable richesse, le travail aliéné

\section{Resumen}

El Aude, un departamento rural en el suroeste de Francia es el sitio de una de las concentraciones europeas más longevos de la práctica contra-cultural. Aquí, pueblos abandonados por el devastador posterior éxodo rural la Segunda Guerra Mundial fueron descubiertos por los radicales entusiastas de la generación del '68 que pudieron establecerse porque la propiedad era barato. De nuestra investigación etnográfica, nos encontramos con que una comunidad receptiva, recursos económicos y un estado de bienestar fuertemente intervencionista proporcionan el terreno fértil en el que el enfoque no normativa practicantes plenitud 'al trabajo y el dinero puede echar raíces, crecer y extenderse. Identificamos un continuo de prácticas económicas alternativas que abarcan una gama de enfoques para trabajar en sí, algunos privilegiando una vida de simplicidad radical y autonomía y otros interesados en el desarrollo de las empresas artesanales con más éxito. Encontramos un lugar para que los neo-medievalistas/ruralistas que un fetiche prácticas como el arado tirado por caballos y los agricultores ecológicos a gran escala. Por último, se muestra que las prácticas de plenitud entre los alternatifs Aude están unidos por la dependencia comunal - por las redes de apoyo y cooperación que se basan en estar entre y cuidar a los demás. Regalar y 'echar una mano' son clave para estas economías, lo que les permite florecer. Si bien esto plantea preguntas sobre el costo de vida para aquellos que no participan por cualquier razón en esta red de intercambio, vemos regalar como fundamental para las economías alternativas duraderas. Este caso refina nuestra comprensión de largos debates alrededor de la necesidad y conveniencia de la autosuficiencia.

Palabras clave: economías alternativas, la dependencia comunal, la agricultura sostenible, Aude, alienación

\section{Introduction}

En l'absence de changement révolutionnaire, jusqu'où les pratiques économiques alternatives peuvent à la fois réduire l'impact de la dévastation écologique, du consumérisme effréné et de l'accroissement des inégalités et permettre de développer un écosystème durable avec des modes vie plus équitables et gratifiants? Quelle est notre capacité à transformer de manière progressive ce système économique mondial, destructeur et instable, que nous appelons capitalisme? Pour comprendre comment changer le monde, il faut non seulement une pensée visionnaire, mais aussi une praxis, ou le développement de la connaissance au travers du processus actif de changement social. C'est dans cette optique que nous avons été attirées par une nouvelle forme d'organisation du travail pratiquée dans l'un des plus remarquables centre européen de production locale, où la production à petite échelle côtoie conscience écologique: le département de l'Aude en France.

C'est là, dans la partie sud-ouest de la France, que des villages isolés, désertés par la population ou frappés par la violence de l'exode rural depuis la Seconde Guerre mondiale, ont été découverts par des radicaux de la génération '68. Longtemps l'un des départements les plus pauvres du pays, les terrains à très bas

\footnotetext{
${ }^{2}$ Dans les traductions du concept de Juliet Schor, la plénitude, le terme 'la véritable richesse' a été utilisé. Alors qu'il est possible d'avoir la richesse sans la plénitude, 'la nouvelle richesse' et 'la véritable richesse' expriment l'idée d'une nouvelle approche de la richesse. Nous utilisons les trois termes de façon interchangeable.
} 
prix de l'Aude ont permis à ces migrants aux moyens modestes de s'établir en petites exploitations et de construire une économie locale autour de l'auto-approvisionnement, de la production à petite échelle et de l'échange, ce qui leur a donné une indépendance inhabituelle par rapport à l'économie dominante et monétaire. Au cours des trente dernières années, la croissance de l'économie alternative et le prix comparativement bas des terrains ont continué à attirer de nouveaux migrants venus de France, des Pays-Bas, d'Allemagne, de Suisse, de Grande Bretagne et de Belgique, avec une conscience anticonsumériste, un désir de simplicité et de durabilité écologique. Alors que de nombreuses anciennes régions fortes de l'économie et de la culture alternative en Europe de l'Ouest ont sombré, les collines rocheuses de l'Aude, et plus particulièrement sa Haute Vallée, ont retenu une masse importante de fermiers et d'artisans alternatifs. L'écroulement du bloc de l'Est et du socialisme d'État a conduit de nouveaux migrants de l'Allemagne de l'Est et de la Pologne à s'y installer dans les années 1990 et 2000, et même si le prix des terrains commence à augmenter, les jeunes alternatifs continuent à affluer dans cette région.

Au travers d'une ethnographie de petits producteurs de fromage, d'huile d'olive, du pain, de la viande, du miel et des légumes biologiques—nous avons cherché à comprendre comment s'est développée l'économie de production durable, d'auto-approvisionnement et d'entraide dans la Haute Vallée et les Corbières, nos deux sites d'études. Qu'est-ce qui a motivé la plupart de ces gens à quitter la vie urbaine pour se recentrer sur des formes de production biologique à petite échelle et à la technologie de base? Dans quelle mesure ont-ils réussi à bâtir une culture commune et une communauté, et à quoi ressemblent-elles? Comment comprennent-ils et négocient-ils leur relation au marché formel et à l'État français?

\section{Les économies diverses: vers une praxis de l'abondance}

Des cas tels que celui-ci sont célébrés par certains chercheurs, mais regardés avec beaucoup de scepticisme par d'autres. Les économistes néoclassiques, tout comme les marxistes, ont tendance à réaffirmer la versatilité et la virilité du capitalisme, le faisant apparaître comme une force inévitable, un créateur de paysages implacable. Mais une zone d'un intérêt croissant démontre que le capitalisme n'est pas une force fatale, un rouleau compresseur qui aplatit le monde, mais un mouvement qui doit être porté-il ne se produit pas par hasard (Deutsch 2010; Tsing 2009), c'est aussi un système qui 'prend l'eau' et dont les pouvoirs sont souvent dispersés (Burke et Shear 2014; Gibson-Graham 1996; Massey 2000). J.K. Gibson-Graham et d'autres ont incité les chercheurs à explorer la multitude des 'économies diverses' en dehors du 'business as usual', c'est-à-dire de la routine capitaliste, sous la forme d'échanges sans argent, d'auto-approvisionnement ou d'approvisionnement communautaire, de circuits de soins et de travail ordinaires non-rémunérés, et de dons informels. Ils suggèrent que ce qui semble faire partie des mêmes vieilles formations capitalistes peut, sur un examen plus approfondi, les économies de soutien, l'éthique et l'écologie qui se écartent de la norme inhumaine, d'exploitation et non durable (Gibson-Graham 2006). En étudiant les caractéristiques de ces économies diverses, leur champ de force interne et externe, et leur viabilité en tant qu'objets de militantisme et de politique, nous pourrons leur donner plus de légitimité dans le débat mondial autour des alternatives au capitalisme (Gibson-Graham 2006). Des chercheurs qui partagent le même point de vue ont commencé à étudier ces formes d'organisations économiques alternatives, du Nord planétaire (global north) aux pays du $\mathrm{Sud}^{3}$, comme les échanges en dehors du marché capitaliste dérivés du troc, groupes d'achat solidaires, ou les systèmes de commerce basés sur l'échange local (voir Fickey 2011; Grasseni 2013; Hughes 2005; Leyshon 2005; Oberhauser 2005).

Les sceptiques mettent en question le potentiel des économies alternatives (Glassman 2003). Immanquablement ils trouvent "que pour chaque coopérative locale avec peu de membres, il existe un WalMart ${ }^{4}$ rempli du sol au plafond de marchandises produites par des processus tout droit sortis des pages du Capital de Marx" (McCarthy 2006, 99). D'autres pointent du doigt l'injection de revenus et de richesses issus

\footnotetext{
${ }^{3}$ L'expression 'global north' ne fait pas seulement référence aux régions géographiques; par exemple, l'Australie fait partie du Global North. Le concept fait référence à une certaine conception de la politique et de la démocratie qui a partie liée avec le système capitaliste impérialiste.

${ }^{4}$ Présent dans 27 pays, Wal-Mart vend une variété de produits ménagers bon marché et les commandes de plus d'un tiers du marché de l'épicerie US. Les équivalents français sont Auchan, Carrefour, et Intermarché. En Grande-Bretagne, Tesco a le même degré de contrôle du marché.
} 
de l'échange capitaliste dans l'économie alternative, le travail domestique et les prestations de soin nonrémunérés des femmes, voire l'asservissement au travail (Lawson 2005).

Cette piqure de rappel concernant les relations de travail est bienvenue-il est certain que la longue histoire de l'esclavage et du travail forcé devrait nous mettre en garde contre toute idéalisation des systèmes économiques hors capitalisme. En cela, il n'est pas mauvais pour ceux qui recherchent une économie diverse de prêter l'oreille au scepticisme des théoriciens de l'économie de résistance, qui nous rappellent que l'opposition classique entre économie capitaliste et économie informelle cache une contigüité de fait. Le capitalisme fait sous-traiter les couches les plus basses de la production mondialisée par l'économie informelle et se repose de plus en plus sur le travail sous-payé ou non-payé dans l'économie informelle pour la subsistance des travailleurs avec de très bas salaires (Portes, Castells, et Benton 1995).

Néanmoins, nous sommes toujours d'accord avec Gibson-Graham pour dire que les visions totalisantes d'un pouvoir capitaliste toujours en expansion et partout adaptable sont teintées de paranoïa et de défaitisme. Interpréter le travail domestique, l'échange informel ou l'économie du don seulement dans la mesure où ils bénéficient au capitalisme masque non seulement les enseignements qu'elles peuvent nous offrir au sujet de l'économie durable et locale, mais dévalorise aussi l'immense créativité, la connaissance culturelle et les connexions humaines qui peuvent être potentiellement transmises et renforcées au travers de telles pratiques.

Á valoriser ces pratiques dans le cadre d'une explication qui tisse une analyse des relations sociales, l'écologie, l'état, et divers processus économiques (capitalistes et non capitalistes) a été la mission de l'écologie politique ${ }^{5}$ (Bebbington et Batterbury 2001; Escobar 1996; Robbins et Monroe Bishop 2008). L'écologie politique a toujours souligné les moyens de subsistance et la vie créées en dépit de, exercées en tandem avec, ou faites en manipulant les forces locales et globales (développement, la race, ${ }^{6}$ l'État coloniales ou postcolonial, le sexe, le capitalisme, le foncier, l'indigénéité). Il ne est pas un grand pas, alors, de relier les idées de Gibson-Graham à l'écologie politique, un effort fait récemment dans une section spéciale dans le Journal of Political Ecology (Burke et Shear 2014). Dans cette section, nous trouvons l'accent sur les pratiques économiques qui sont semblables à ceux trouvés dans l'Aude (par exemple des villes de commerce équitable (Lyon 2014), et les groupes de solidarité d'achat (Grasseni 2014)). D'autres exemples de l'écologies politiques 'plus-que-capitaliste' se concentrent, comme nous faisons, sur les subjectivités économiques. Par exemple, dans le cas de la pêche du nord-est USA, Kevin St. Martin discute de l'importance de la coopération et de la dépendance mutuelle dans les "subjectivités communautaires" de pêcheurs (2005, 973). Lucy Jarosz fait valoir contre l'idée que une éthique de soins est toujours néolibérale, exprimées chez les femmes qui pratiquent l'agriculture urbaine et l'approvisionnement alimentaire (Jarosz 2011). Pour Anna Tsing, la question ne est pas 'capitalocentrisme' vs. le 'non-capitalisme', mais la nécessité urgente de comprendre la diversité des espaces, subjectivités, écologies et les possibilités d'action radicale dans certains niches, caché dans les chaînes d'approvisionnement mondiales. Ce qu'elle voit dans son exemple de la ramassage des champignons matsutake, qu'elle voit comme un performance de l'identité culturelle ainsi que d'une activité économique (Tsing 2012, 45; 2009, 171). De même, dans les "collectifs anarchiques rigoureusement organisés" (Grasseni 2013, 109) qui sont groupes d'achat solidaires Italiens, Cristina Grasseni trouve pas une issue du capitalisme tout à fait, mais plutôt "un effort laborieux, réflexive et collective de transformer les secteurs [du capitalisme] dans les économies de confiance, dans dont le respect réciproque, de solidarité et de co-production déplacent la pratique économique loin de la seule considération de maximisation du profit" (Grasseni 2013, 29). Nous espérons que nous pourrions retrouver notre cas à proximité de ces exemples.

L'assaut du capitalisme sur les écosystèmes du monde est à l'origine de la création d'économies alternatives dans le Nord planétaire. La planète est en danger, avec une croissance de personnes à travers le monde aspirant qui voudraient consommer autant que le Nord planétaire (Zakaria 2011). Depuis plusieurs siècles l'humanité a été avertie qu'elle était sur le point d'atteindre ses limites (Meadows et al. 1972), et cette

\footnotetext{
${ }^{5}$ L'écologie politique' est utilisé en France de se référer généralement à l'environnementalisme. Dans la littérature scientifique anglophone, 'political ecology' est une sous-discipline compris comme tel en géographie, anthropologie, études de développement et les études environnementales.

${ }^{6}$ Nous définissons la race comme un "assemblage complexe des phénotypes et des environnements réarrangé par le colonialisme et le capitalisme ... [et] le matériau et la division mentale des organismes en groupes selon déplaçant critères" (Saldanha 2011, 453). Race est réel dans le sens que, partout dans le monde, les gens attachent systématiquement la valeur à phénotype (Saldanha 2006).
} 
façon de voir les choses est maintenant aggravée par l'extinction de la biodiversité planétaire et le changement climatique. Cette menace de destruction imminente requiert que l'on se serre la ceinture, même si la majorité n'a jamais souffert de 'l'affluenza. ${ }^{7}$ Mais nous nous doutons que des récits à base de pénurie et de frugalité sont regarder avec la même d'enthousiasme par la majorité. En lieu et place de cela, mobiliser les gens en faveur d'économies politiques différentes demande une réorientation et un enrichissement des cadres de 'suffisance' contemporains-se concentrer moins sur la pénurie que sur le plaisir, la communauté et les types d'abondance alternative (voir aussi Collard, Dempsey, et Sundberg 2015; Haraway 2014). En fait, ces dernières notions reflètent très bien les intentions des alternatifs et des artisans dont nous parlons dans cet article.

Il s'agit là du projet de Juliet Schor, La véritable richesse (2010), qui démontre le potentiel révolutionnaire des organisations ascendantes (bottom-up) à petite échelle qui se désolidarisent du capitalisme routinier. Elle souligne le besoin de rejeter un matérialisme quantitatif en faveur d'une vrai matérialismedans le sens de l'engagement passionné avec la matérialité du produit, son histoire sociale et naturelle, et son futur en termes de la durabilité. Les 'nouvelles répartitions du temps', 'l'auto-approvisionnement' et 'le réinvestissement dans la communauté et nous-mêmes' sont tout aussi importants. ${ }^{8}$

Cet article divise notre cas d'étude en éléments similaires, mais il souligne les subjectivités et les relations politiques au sein desquelles s'intègre l'expérimentation économique. Nous démontrons qu'a diminuer la création de formes capitalistes de production et d'échange demande non seulement une économie alternative consciente d'elle-même, mais aussi une critique collective des formes économiques dominantes et des hiérarchies sociales. L'économie alternative vitale de la Haute Vallée, en particulier, est inscrite dans une vision globale et une culture du changement qui réoriente fondamentalement les notions de travail et de production, de richesse et de sécurité.

\section{Notre argument}

L'article avance quatre autres arguments d'intérêt général pour l'étude des économies alternatives. Premièrement, notre cas démontre l'importance cruciale d'introduire les pratiques économiques alternatives dans des conditions socio-économiques favorables. L'Aude était assez bon marché pour permettre aux néoruraux de s'installer en grand nombre et à se pérenniser grâce à une ténacité qui a soutenu des vagues successives d'immigration. La bonne volonté de nombreux Audois plus âgés pour accepter et guider ces migrants a été tout aussi importante.

Deuxièmement, nous défendons l'idée que les pratiques économiques alternatives ne doivent pas exister dans un espace raréfié, pur dans lequel 'a gagner sa vie' reste subordonné à un idéal. Nous voyons plutôt un continuum d'approche du marché et du travail lui-même, des alternatifs radicaux qui privilégient la simplicité foncière et l'autonomie à ceux qui proposent des entreprises artisanales avec des produits de haute qualité. Ils peuvent même aller jusqu'à faire du commerce avec une boutique de San Francisco ou un restaurant de trois étoiles. Il y a cependant beaucoup d'imbrications entre ces deux orientations; il faut souligner que tous les producteurs rejettent explicitement certains éléments centraux du capitalisme traditionnel, particulièrement l'idée de prioriser le profit par rapport au produit et l'asservissement au travail. Ce qui importe le plus c'est la résolution à vivre d'une manière relativement simple et de trouver une gratification non pas dans le profit, mais dans ce qu'on fait les caractéristiques éthiques, communautaires et esthétiques de la production sont des fins en elles-mêmes.

Troisièmement, les pratiques économiques diverses des alternatifs de l'Aude sont soutenues par des réseaux serrés de soutien et de coopération. Le grand nombre de dons, de coopérations et d'échanges informels que nous analysons dans notre étude constituent une sorte d'approvisionnement communautaire qui est essentiel à la grande qualité de vie et à la survie économique des petits producteurs et des alternatifs de la région, et qui abaisse substantiellement le coût de la vie. Nous voyons donc une forte synergie entre l'approvisionnement domestique et la vitalité communautaire en général, car les réseaux d'échange informel

\footnotetext{
${ }^{7}$ Il s'agit d'un jeu de mot sur 'affluence' et 'influenza', qui veut dire 'la grippe' en anglais.

${ }^{8}$ En utilisant ce cas d'étude, nous abordons en plus grand détail le travail de Schor.
} 
fournissent simultanément un soutien matériel essentiel aux ménages tout en créant et renforçant des liens sociaux forts.

Quatrièmement, les petits producteurs de l'Aude ont grandement bénéficié de l'interventionnisme de l'État français. Sans une variété de programmes pour les nouveaux fermiers et l'agriculture biologique, la préservation des terres agricoles, l'assurance medicale, la sécurité sociale et les allocations familiales, les ressources modestes et les arrangements faiblement rémunérés de plusieurs de nos interviewés les auraient laissés dans une précarité vulnérable et peut-être moins soutenable, faisant de cette économie alternative un projet nettement moins inclusif. En somme, une communauté réceptive, des ressources de base peu coûteuses et la protection sociale ont contribué à créer ce sol fertile où des approches non normatives du travail et de l'argent ont pu prendre racine, se développer et se propager.

\section{Le paysage social du site de la recherche et les méthodes}

Notre étude a bénéficié du grand nombre de connaissances et d'amitiés de Teresa dans la région de l'Aude - sa mère a habité pendant plus de vingt ans une petite ferme des Corbières et Teresa a passé plusieurs mois dans la Haute Vallée chaque été ces huit dernières années. La plupart des producteurs étaient déjà des amis ou des connaissances. Nous savions donc déjà que nos deux sites occupaient une place quelque peu différente dans l'économie alternative de l'Aude. La Haute Vallée, qui est un centre 'hippie' et 'écolo' avec de nombreux migrants étrangers, est bien connue des réseaux de la contre-culture européenne. La région de Lagrasse dans les Corbières, plus liée à la monoculture vinicole traditionnelle de la région, a aussi attiré des soixante-huitards et des migrants plus récents cherchant à vivre différemment, avec une concentration un peu plus forte de ceux qui se définissent comme artisans.

Le tournant qu'a été mai 1968 a eu un effet subtil mais indélébile sur le caractère de l'Aude, quand les migrants de la contre-culture en France, aux Pays-Bas, en Belgique, en Grande Bretagne, en Allemagne et en Suisse urbaine ont rempli au goutte à goutte ses villages isolés, et même ses fermes et ses granges abandonnées les plus isolées. Avec leur enthousiasme pour expérimenter des modes de vie radicalement différents, ce groupe a eu tendance à s'identifier comme 'alternatifs'. Quelques résidents plus anciens, qui ne partagent pas les mêmes envies radicales, n'arrivaient pas à comprendre pourquoi quelqu'un pouvait choisir le dur labeur de l'existence paysanne et, en effet, trouvaient le mode de vie et les motivations des 'néo-ruraux' déconcertants.

Les arrivants ont redonné vie à la culture publique de l'endroit, défiant les mœurs traditionnelles tout en faisant montre des nouvelles possibilités économiques de la région. La première génération n'a jamais vraiment appartenu aux communautés fermées des villages, mais leur plus grand nombre dans la Haute Vallée a généré une culture alternative plus forte, et, dans les cas où ils ont pris possession de hameaux et de villages clairement abandonnés, ils se sont rapidement impliqués dans la politique locale. ${ }^{9}$

Les vagues suivantes de migrants ont continué le travail intense de reconstruction des fermes en ruine et ont commencé à gagner leur vie, accompagnés par le retour des enfants des premiers alternatifs. Beaucoup ont choisi l'Aude parce qu'elle était plus abordable qu'une Provence de plus en plus chère au nord-est.

En gros, les producteurs de la Haute Vallée ont développé un mode de vie beaucoup plus alternatif que leurs homologues du nord dans les Corbières. Au travers de cette étude nous avons trouvé un grand éventail d'orientations vers la production et la politique de l'agriculture bio. Alors que certains étaient impliqués dans la production et la distribution à grande échelle, la plupart, qu'ils soient artisans ou alternatifs, ont gagné leur vie avec les gains de leurs petites ventes sur un ou deux marchés, prenant la décision de ne pas étendre leur entreprise ou mettre leurs concurrents au chômage.

Pendant l'été et l'automne 2011 nous avons réalisé 22 entretiens avec 18 petits producteurs individuels ou en couple, deux anciens producteurs et deux militants locaux. Pour les besoins de cette étude nous avons défini les petits producteurs comme des gens a) qui vendent sur un marché local, dans les magasins de producteurs et/ou les coopératives, et b) qui ne vendent pas aux grands distributeurs parce que ça ne correspond pas à leur modèle d'affaires ou leur position idéologique. Nous nous sommes aussi impliquées dans l'observation des participants sur les marchés, dans les coopératives, dans une foire biologique ${ }^{10}$ et une Boutique Paysanne. Teresa s'est portée volontaire régulièrement à la coopérative 'Amaranthe' d'Espéraza

\footnotetext{
${ }^{9}$ Voir aussi Cortes-Vazquez (2014), le cas de Cabo de Gata en Andalusia, Espagne.

${ }^{10}$ La Foire Bio de Couiza est organisée par l'OGN Nature et Progrès.
} 
pendant 9 mois à partir d'octobre 2011. Pour discuter de nos conclusions, nous avons organisé deux groupes de discussion, un à chaque site, au cours de l'été 2013. Nous avons collecté nos observations au travers de notes prises sur le terrain et de transcriptions d'entretiens, concentrant nos analyses sur les thèmes explorés cidessous.

Les marchés étaient des sites ethnographiques particulièrement importants. Comme dépositaires des symboles de la France 'traditionnelle', les imaginaires des marchés connectent l'identité française à la vie rurale et aux produits locaux qui incarnent un climat, un sol et un terroir spécifique, bien que la réalité soit assez différente (Buller 2003; Demossier 2003; Fantasia 1995; Hervieu et Purseigle 2008). Chaque semaine des marchés en plein air persistent dans l'âge postindustriel, même s'ils sont devenus moins importants pour la vie publique et l'approvisionnement domestique dans les villages tout comme les villes.

Le marché urbain de Lézignan est large, une mixture diverse de producteurs locaux et de vendeurs de fruits et légumes qui partagent l'espace avec des vendeurs de vêtements, de chaussures et d'ustensiles à bas prix. Le petit marché de Lagrasse se situe à l'opposé. Rempli presque exclusivement de petits producteurs locaux, il fournit des produits à la population locale tout au long de l'année mais ses sept ou huit stands réguliers doublent leur nombre lorsque la clientèle gonfle avec les propriétaires de maison de campagne et les touristes pendant l'été. Nous nous sommes entretenues avec neuf personnes d'âges et d'origines nationales différents autour du canton de Lagrasse dans les Corbières. Le marché de Lagrasse est trop petit en lui-même pour soutenir les producteurs de la région, c'est pourquoi la plupart vendent au plus grand marché de Lézignan à l'est. Nous avons recruté un deuxième panel de neuf producteurs sur le marché le plus important de la Haute Vallée dans la petite ville d'Espéraza. Ici aussi nombre de producteurs vendaient sur les marchés à Limoux au nord, Mirepoix dans l'Ariège à l'ouest et sur un plus petit marché dans le village voisin de Quillan (Figure 1).

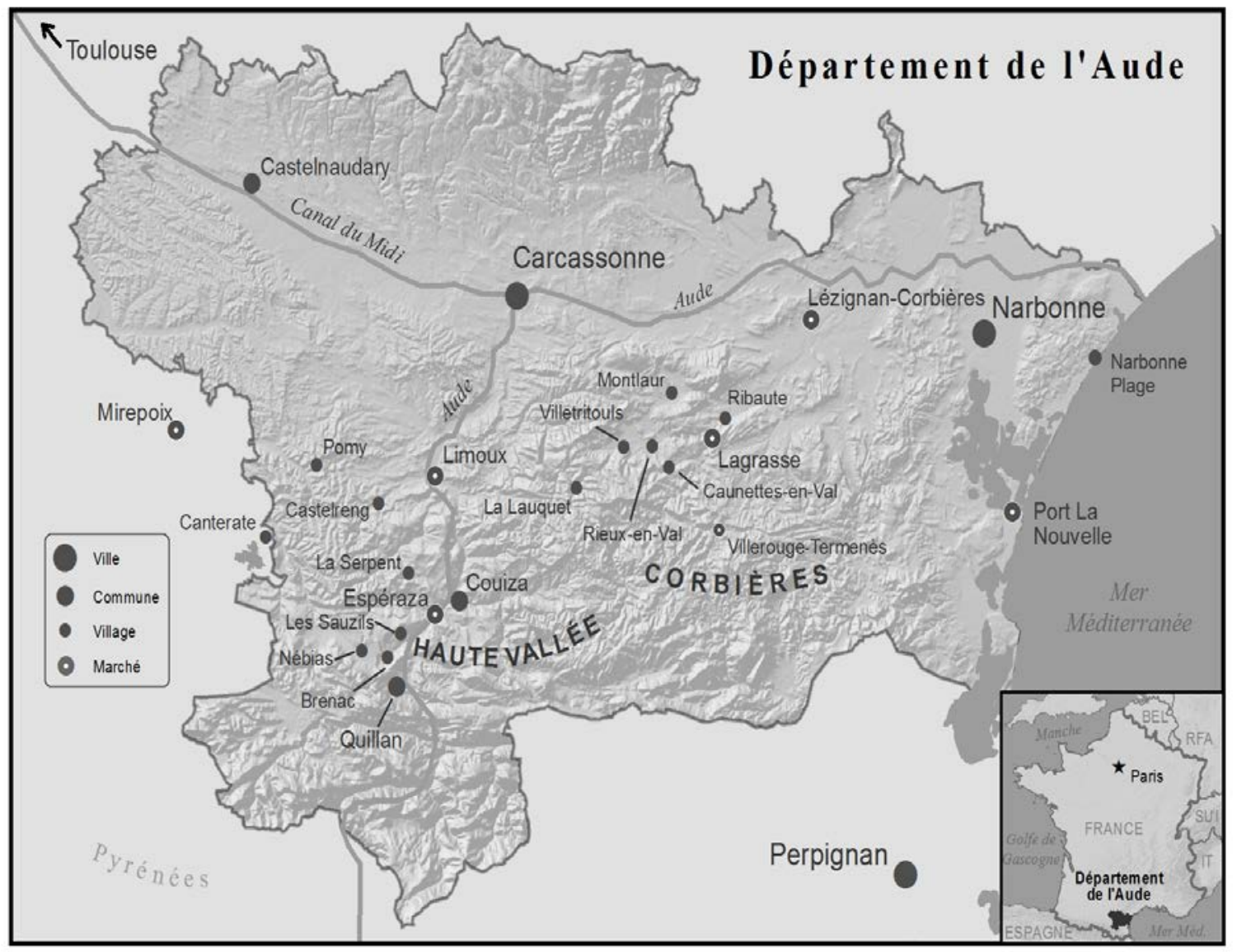

Figure 1: Carte des sites de recherche dans l'Aude. Cartographe: Birgit Muehlenhaus 


\section{Sur le terrain}

\section{Lagrasse et les Corbières}

La région Lagrasse dans les Corbières se trouve à environ trente kilomètres au sud-est de Carcassonne, où les collines rocheuses recouvertes de broussailles entourent des vallées toujours dominées par la culture vinicole à petite échelle (Figure 1). A deux heures de la mer, c'est une terre des vents violents-le Cers sec venant de l'ouest et le Marin méditerranéen humide—souvent mentionnés comme étant l'une des grandes difficultés de la vie dans l'Aude. Les odeurs de thym, romarin et fenouil, particulières à la méditerranée, imprègnent l'air sous un soleil qui brûle un sol ayant résisté à des centaines d'années de culture vinicole. Comme de nombreux ruraux du sud, la plupart des habitants des Corbières vivent toujours dans des villages et des petites villes de 50 à 800 personnes. Des avenues de platanes cèdent le terrain à une masse compacte de vieilles maisons enchevêtrées dans des petites rues, maintenant accompagnées de quelques villas plus modernes sur les côtés.

Bien qu'elle ne ressemble en rien à la vie isolée des Corbières d'avant la seconde guerre mondiale, la vie de village a toujours l'air profondément renfermée sur elle-même. En dehors du service scolaire il n'y a pas de transports publics. Le commerce est limité aux villages plus importants et les personnes plus âgées qui ont un accès limité aux voitures comptent sur les visites régulières des camionnettes vendant de la viande et du poisson, ramenant les produits des bouchers et des poissonniers de Carcassonne. Ces sources uniques de nourriture perdent lentement du terrain par rapport aux supermarchés.

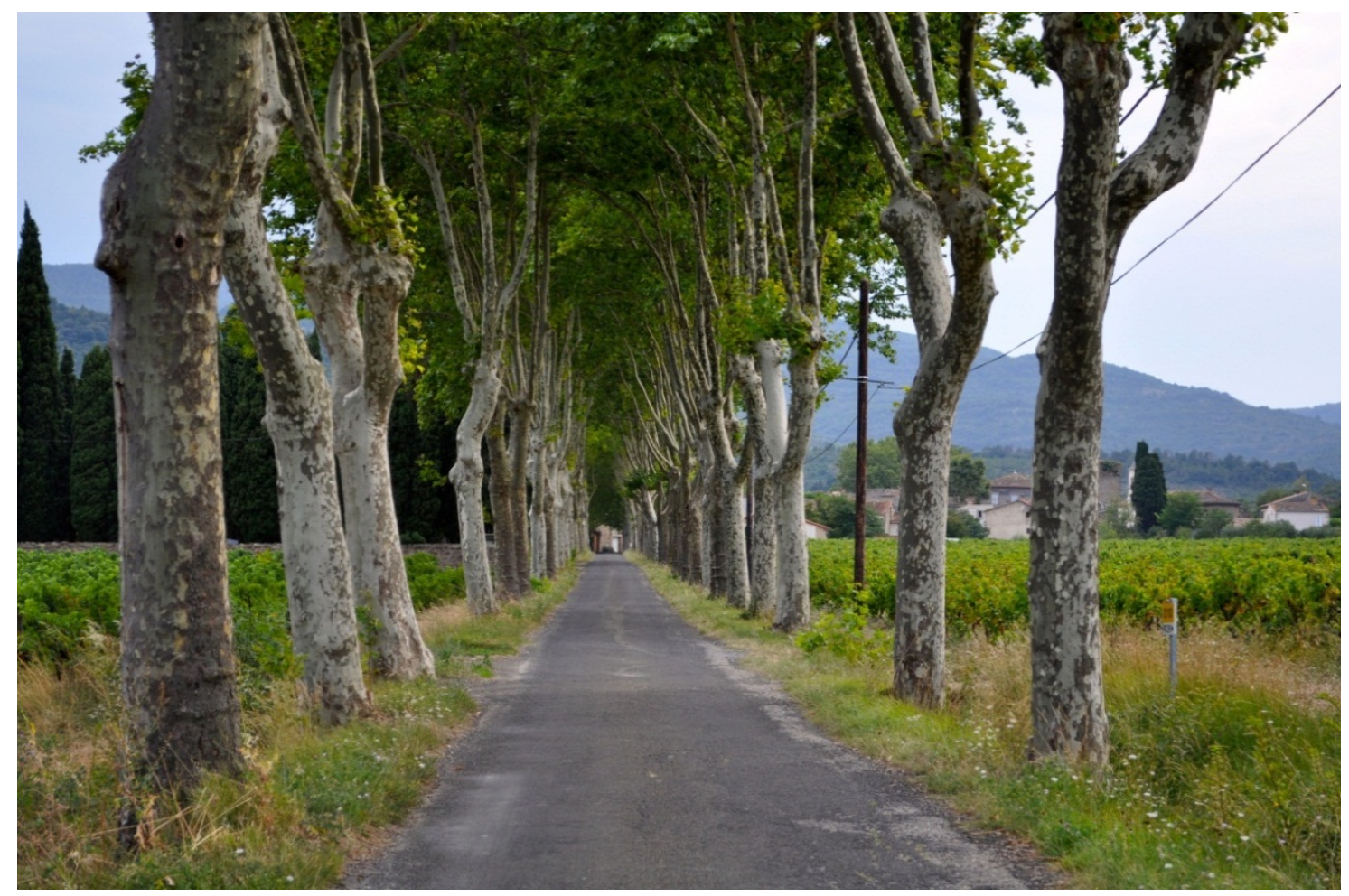

Figure 2: Vignes de chaque côté de l'entrée à Rieux en Val, Val de Dagne, Corbières. Photo prise par Rachel Slocum.

Reconnue pendant des siècles comme une région vinicole, les vieux vins se sont enracinés solidement dans la vie culturelle et économique de l'Aude, si bien que les nouveaux arrivants se sont souvent sentis littéralement et figurativement "coincés par les vignes" (Figure 2). Alors que la production vinicole a empêché 
ces villages de se vider comme dans la Haute Vallée, sa domination a entravé les économies alternatives. Les vins ont toujours eu la priorité pour les meilleurs terrains, occupant les endroits proches des ruisseaux et des rivières qui pourraient servir d'autres fins, comme le jardinage:

Mais avant il y avait beaucoup plus de vignes qu'aujourd'hui, et donc nous étions coincés par les vignes... C'est le vin, après un peu l'huile d'olive, récemment, car l'huile d'olive ça a 15 ans, il faut pas croire que ça date. Enfin ça date de très longtemps l'huile d'olive mais les vignes avaient pris toute la place. La monoculture de la vigne a étouffé toutes les autres cultures.

Alors que les champs d'oliviers représentaient jadis une grande partie de l'économie, ils ont subi une gelée dévastatrice en 1956 qui en a détruit plusieurs. Selon une histoire qui court parmi les alternatifs, l'esprit rural conservateur (ou peut-être les coopératives vinicoles) a inventé un nouveau dogme-la région ne pouvait pas soutenir une production commerciale d'olives. Un demi-siècle plus tard, les oliveraies sont réhabilitées par les nouveaux arrivants, conseillés par quelques-unes des personnes plus âgées qui avaient continué à les cultiver.

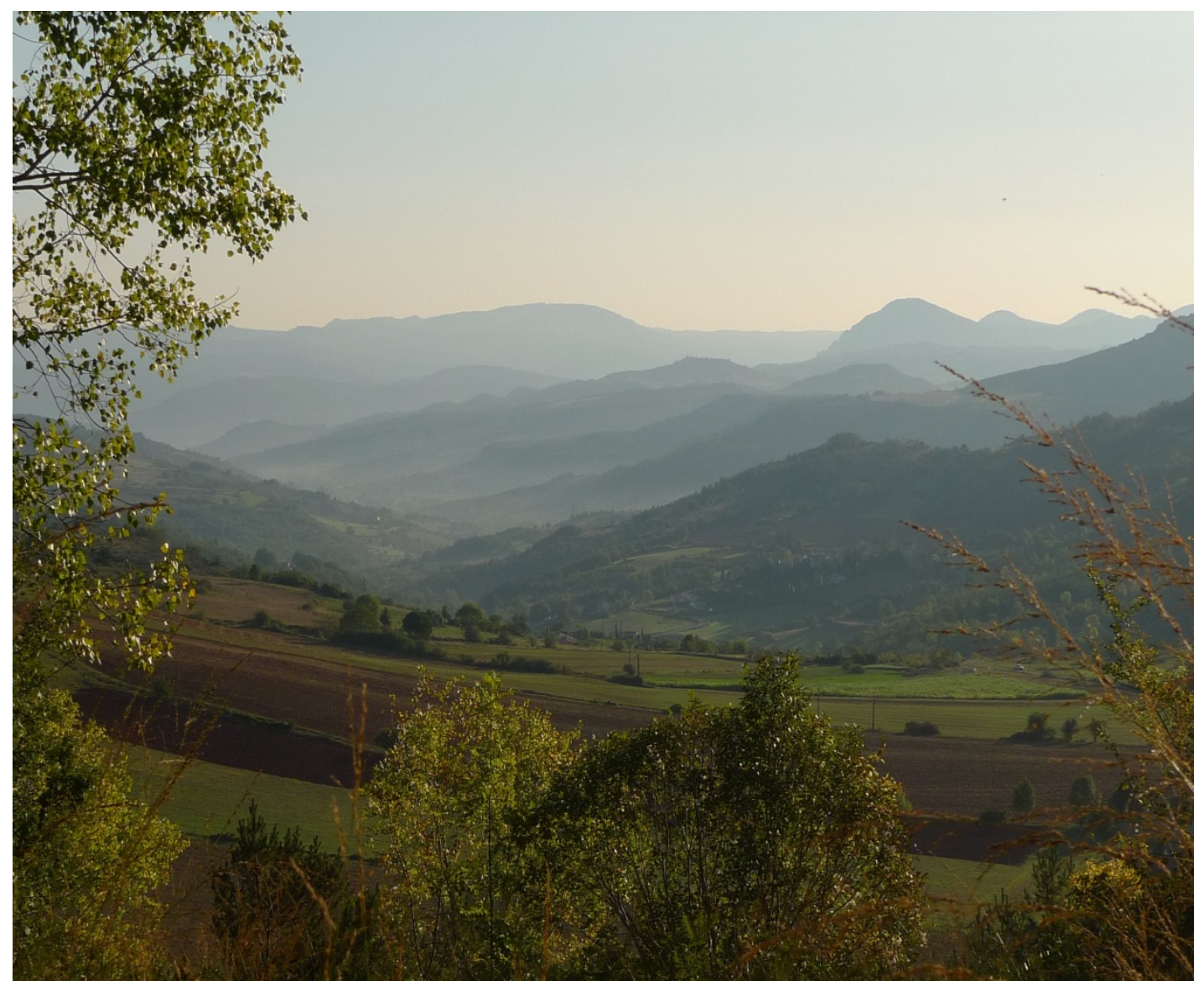

Figure 3 : Paysage de la Haute Vallée à Brenac. Photo prise par Teresa Gowan.

\section{La Haute Vallée}

Si vous conduisez au sud-ouest de Lagrasse pendant environ 50 kilomètres, les interminables virages des Corbières vertes vont finir par vous emmener à la Haute Vallée de l'Aude, où des collines boisées et isolées plongent et grimpent dans les vallées profondes et les falaises imposantes de l'est des Pyrénées (Figure 3). 
A l'exception d'un amas de petites villes situées près de la rivière Aude, l'exode rural de la Haute Vallée a été extrêmement sévère, laissant de nombreux hameaux abandonnés et d'autres villages avec seulement une poignée d'hommes et de femmes vieillissants. Au final, au travers des années '70 et même au début des années '80, les vieilles maisons de village en pierre pouvaient être achetées pour une bouchée de pain, attirant une masse cruciale de soixante-huitards pressés de quitter la ville industrielle pour trouver l'air pur et un mode de vie plus 'naturel' (Cazella 2001). Les relations sociales n'étaient pas toujours faciles. Les identités villageoises localisées faisait que même quelqu'un venant de la vallée la plus proche était considéré comme un étranger. Les Français tout comme les nouveaux arrivants venus de l'étranger ont dû faire des efforts pour être accepté par la population locale qui rechignait même à vendre du terrain qui n'avait pas vraiment été utilisé depuis des dizaines d'années. Il reste des tensions entre la population locale et les nouveaux arrivants, tournant souvent autour de disputes concernant la chasse, traditionnellement pratiquée dans tous les terrains agricoles et forestiers, indépendamment de la propriété. Il était evident que si quelqu'un cherche à louer ou à emprunter un terrain plutôt qu'à l'acheter, la chance d'être accepté est plus grande.

Cependant, compte tenu le dommage énorme effectuée par l'exode extrême, certaines personnes âgées étaient prêtes à consentir à l'immigration de jeunes voisins vigoureux, en dépit de leur comportement bizarre. Fermier et boulanger, Patrick nous dit qu'il avoir toujours maintenu de bonnes relations avec les gens du coin, même en tant qu'il était membre d'une communauté campant dans un hameau en ruine. Et le fermier Dédou nous a dit à quel point il avait été émerveillé d'avoir été si chaleureux accueilli quand il avait été embauché comme jeune berger dans les années '60:

Les personnes âgées [celles qui sont restées au village] étaient heureuses de nous voir arriver, parce que je crois qu'elles étaient déprimées de voir tous les enfants partir. Oui, elles étaient heureuses car elles disaient "mon fils a désormais une meilleure situation", mais au fond d'elles-mêmes il y avait une véritable tristesse de voir leur terrain abandonné, leur ferme négligée.

Aujourd'hui la Haute Vallée est toujours un endroit insolite. Pour commencer, certaines exploitations sont minuscules; avec quelques marchands vivants dans de rudimentaires maisons en paille ou des yourtes sur un lopin de terre, apportant un petit assortiment de légumes variés au marché. Le purisme néo-médiéval qui caractérisait beaucoup d'anciennes communautés n'est plus la norme, mais l'extrême simplicité est toujours aussi influente.

Un commentaire, très suggestif du caractère hors du commun de la Haute Vallée dans l'économie des petits producteurs français, vient de Suzanne, une productrice à la retraite issue d'une autre région de France, qui ne pouvait pas croire à quel point de nombreux petits producteurs de la Haute Vallée pouvaient être inefficaces et peu fiables.

C'est incroyable, c'est comme s'ils ne s'intéressaient pas du tout à l'argent. Ils veulent seulement être 'baba cool' et faire ce qu'ils ont envie de faire, spontanément... Oui, ils travaillent, mais ils ne sont pas prêts à être efficaces. Ce n'est pas comme ça dans d'autres domaines. La Haute Vallée, c'est vraiment quelque chose d'autre!

Elle a rigolé et roulé ces yeux.

Notre travail sur le terrain et nos entretiens ont confirmé l'impression de Suzanne, que la culture de la Haute Vallée donnait moins d'importance au rendement ou aux revenus élevés. Dans bien des cas cette orientation différente était explicitement articulée: il y avait une forte volonté anticapitaliste chez plusieurs des personnes interrogées et d'impressionnants graffitis ornaient les route principales de la région avec des logos de syndicats et de slogan du type 'Le monde n'est pas une marchandise.' La transformation du logo Shell en 'hell' est restée intacte depuis plusieurs années dans la rue principale d'Espéraza (Figure 4). 




Figure 4: Espéraza (S)hell, Haute Vallée, photo prise par Teresa Gowan.

\section{La vrai matérialisme}

\section{La commercialisation holistique}

A l'heure de notre arrivée au marché de Lézignan un mercredi d'août à 9h du matin, il y avait déjà dix personnes qui faisaient la queue pour acheter le fromage de Chantal, qu'elle coupait et emballait tout en discutant rapidement et joyeusement avec les habitués et les nouveaux clients. Elle vend rapidement tout son yaourt frais et sa brousse, sa tomme mature et sa tommette sont tout aussi populaires.

Il y a à peu près quarante ans, le mari de Chantal, Jean-Gabriel, a décidé de poursuivre le rêve de sa vie-devenir éleveur ovin. Il est allé à l'école, a travaillé pendant quatre ans, puis ils ont cherché un endroit pour établir leur propre ferme, "arrivant dans l'Aude par chance." Ils n'étaient pas assez riches pour acheter du terrain mais ils ont eu la chance de rencontrer des gens qui leur ont loué du terrain dans la garrigue pour un fermage de huit ans. ${ }^{11}$ Afin d'obtenir des subventions agricoles de la France et de l'Europe, il fallait avoir environ 22 hectares, ce qui voulait dire qu'il fallait trouver une vingtaine de propriétaires terriens prêts à leur octroyer un fermage dans le but d'atteindre le nombre requis. Faisant écho au récit de Dédou, elle nous dit:

Quand nous sommes arrivés ici, il n'y avait que les gens simples qui voulaient bien nous donner des terres. Ils savaient qu'on avait besoin de terres. [Ils disaient] "J'ai un hectare, madame, je ne sais pas où c'est, mais je veux bien te le prêter, pour que tu puisses travailler!

\footnotetext{
${ }^{11}$ Le fermage a été créé pour protéger les personnes qui cultivent le terrain car une fois que le terrain a été cédé sous ce type de contrat il ne peut être résilié qu'avec un préavis de 18 mois. De plus, si le contrat est annulé cela doit être fait dans le but de cultiver le terrain ou de le transmettre aux enfants ; il ne peut pas être retiré pour être vendu.
} 
Chantal et Jean-Gabriel n'appréciaient pas les formes industrielles de production et ils ne voulaient pas non plus s'agrandir. Au lieu de cela, heureusement, ils ont entendu des gens faire leur propre fromage de brebis bio dans la Montagne Noire voisine. Alors que d'autres Audois considéraient ces fromagers comme des 'hippies' plutôt que des producteurs sérieux, pour Chantal les fromages "c'était extraordinaire, vraiment..."

Donc on est allé chez eux, on a goûté leur fromage, et ça a été pour nous la révélation. Donc quand on a goûté ça, on s'est dit avec mon mari: "C'est ça qu'on veut faire". Et donc c'est une autre aventure qui s'est mise en place, car moi j'ai eu la chance d'avoir fait des formations fromagères, plusieurs, peut-être 5 ou 6.

Les petits producteurs s'investissaient passionnément dans la qualité des produits qu'ils fabriquaient et dans la durabilité de leur pratique-que ce soit les chaussettes en laine et les agneaux d'une petite bergerie, les légumes non-traités d'un jardin maraîcher, du miel et des produits à base de cire d'abeilles, ou du fromage de chèvre et du yaourt au lait de chèvre. Ils nous racontaient comment la qualité de leurs produits dérivent de la simplicité- de l'herbe non-contaminée mangée par les moutons de Mireille et Dédou Babeuf à la "magie élémentaire" de la cuisson racontée de manière très évocatrice par chacun des boulangers de la Haute Vallée (Figure 5). Les boulangers Jan et Rolf font tous les deux pousser leur propre blé, alors que les apiculteurs Mireille et Philippe vont jusqu'à prendre la peine de collecter le miel d'une seule origine. Cet accent sur le caractère simple et authentique de la production artisanale est l'exemple type de ce que Schor appelle le vrai matérialisme.
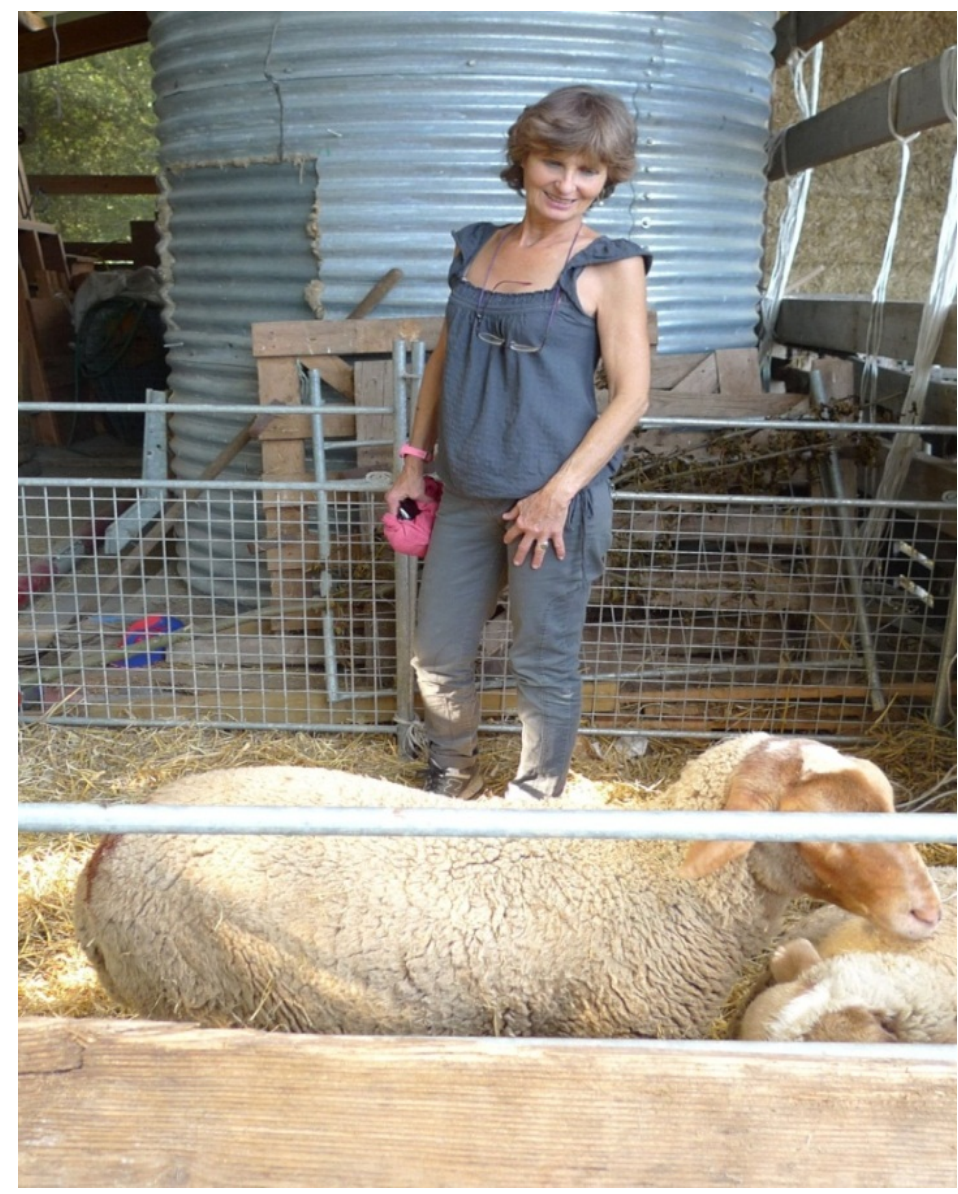

Figure 5: Mireille Baudeuf, photo prise par Teresa Gowan. 
Chantal a compris que la clef était de s'occuper de tous les aspects du produit, de l'origine jusqu'à la vente:

Hier je regardais à la télévision une émission sur le crottin de Chantignole. C'est une des formes typiques du fromage de chèvre. Et les producteurs comme moi font du fromage frais, et le vendent à un fromager qui l'affine. Eux ne font que du fromage frais. Ils ne l'affinent absolument pas. Et c'est dommage. Car la valorisation de leur travail est faite par l'affinage et la commercialisation d'un produit fini. Et je pense que si on est trop gros on ne peut pas faire ça, et si on est à notre échelle, on peut gérer ça.

Chantal (Figure 6) a plus tard renforcé son argument en faisant référence à une femme qui avait récemment commencé à vendre de l'agneau directement de sa ferme dans le Val de Dagne:

C'est extraordinaire ici de ne pas vendre à la coopérative, d'aller à l'abattoir avec ses agneaux et de livrer les gens. Alors eux c'est de l'agneau, les autres c'est du veau, voyez le produit finit. Aller jusqu'au bout de la commercialisation. C'est la seule façon pour nous en petite production de pouvoir se développer, de pouvoir se maintenir.



Figure 6: Chantal Donnet, marché Carcassonne. (c) Jennifer Greco Normandy, utilisée avec la permission.

Des commentaires similaires venant de Micias et Marie liaient la fierté du produit au besoin d'avoir de bonnes relations avec les clients, ces fervents alternatifs faisaient un sirop avec de la lavande et de la menthe qu'ils cultivaient dans les collines au-dessus de Lagrasse (Figure 7): 
On a commencé vraiment tout petit et c'est vrai que d'un coup on a eu de la demande, les gens ils ont dit "ah, c'est génial" et donc, plein de demande, et nous, on n'a pas réussi à suivre au niveau de la production. Donc maintenant on a organisé la production... Maintenant les gens recherchent vraiment ça (les produits des petits producteurs), les gens nous encouragent, "ah bravo! continuez à faire ce que vous faites ”... Les gens ont besoin de contact, de voir le producteur, de discuter avec le producteur...



Figure 7: La lavande chez Micias et Marie Saillard, Corbières. Photo prise par Rachel Slocum.

Avec Jan et d'autres dans la région, ils ont acheté une vieille ferme de pierre où ils vont installer un laboratoire pour sécher les herbes et faire du sirop, préférant, comme Chantal, suivre le développement du produit du début jusqu'à la fin.

Mireille et Philippe, les producteurs de miel, nous ont dit qu'ils prenaient le processus de commercialisation très au sérieux, faisant l'effort d'interagir avec les clients et de chercher de nombreux points de vente. Dans le sud, disent-ils, il y a une certaine honte à faire du miel, une honte qui fait obstacle au type de relation que les gens cherchent à instaurer avec les producteurs. "Tout le monde est bienvenu pour acheter 
notre miel" nous dit Philippe. En contraste, Mireille nous a décrit une potière dont les pots extrêmement chers étaient admirés de tous: le vendeur passait son temps à ignorer les clients et puis elle se plaignait qu'elle ne gagnait pas assez d'argent. Etre alternatif ne devrait pas vouloir dire être en échec au niveau commercial—"tu dois vouloir vendre ton produit" insistait Mireille, exprimant une certaine admiration pour le sens des affaires de Micias et Marie et d'autres producteurs voisins. Mireille et Philippe ont co-organisé le collectif Extraterrien, une façon essentielle de pérenniser leur marché, qui arrange des petites visites dans les fermes du coin (le tourisme fermier) et organise cinq festivals par saison avec du vin local, des produits en cuir, du savon au lait d'ânesse et toute une variété de nourritures.

Chez les clients la production artisanale génère un intérêt dans le travail investi et du respect pour ce qu'il produit, ceci en grand contraste avec la consommation excessive de produits anonymes encouragée par les 'offres spéciales' et les ventes par lots. Un appétit pour le légume 'connu' est évident dans le récit d'Isabelle: "On a beaucoup... de clients chez nous qui sont des gens ...du troisième âge, beaucoup de personnes âgées, ...parce que c'était comme leur jardin avant, nos produits..."

Les interactions directes dans l'environnement sympathique du marché créent des liens sociaux en développant des relations de confiance et d'engagement, et dans ce processus le pain, les oignions, le fromage de tous les jours se trouvent ré-enchantés, prenant une valeur beaucoup plus grande pour le consommateur et le producteur lui-même (Hinrichs 2000; Thompson et Coskuner-Balli 2007). Dans nos groupes de discussion, les producteurs ont souligné qu'une partie essentielle de ce vrai matérialisme était le plaisir qu'ils ont obtenu de leur interaction avec les clients. Alors que des conceptions plus arbitraires de la qualité et des imaginaires nostalgiques peuvent jouer un rôle (Holloway et Kneafsey 2000), la concentration des ventes directes vers un produit dans lequel on a confiance élève ce dernier au-delà du réseau financier délocalisé, créant un matérialisme connu et situé en directe opposition au 'c'est moins cher!' des supermarchés (voir aussi Gilg et Battershill 1999).

Notre cas d'étude suggère que le sens de l'engagement va dans les deux sens. Les clients développent une loyauté vis-à-vis de leurs marchands réguliers, qui en retour font de leur mieux pour garder les prix bas. Mireille et Philippe, par exemple, ont fait payer le même prix pour leur miel au cours des six dernières années, et la plupart des autres producteurs ont parlé de se battre pour garder les prix bas pendant la récession.

L'importance de la confiance et de la valeur par rapport au 'moins cher' est particulièrement vraie dans le cas de l'agriculture biologique vers lequel nous nous tournons maintenant.

\section{Une relation bio enchantée}

Les producteurs de légumes biologiques, Isabelle et Daniel, ont connu leurs premiers clients le jour où Isabelle avait oublié de mettre le panneau 'Bio' au marché de Lézignan. En ce temps-là, les gens avaient peur d'acheter de la nourriture bio parce que le ministère de l'agriculture française suggérait qu'elle était peut-être dangereuse pour la santé. Aujourd'hui la plupart des clients achètent leurs produits parce qu'ils sont bio.

Trente ans après qu'Isabelle et Daniel ont initié leurs clients à la nourriture bio, la certification est venue renforcer une confiance allant au-delà des interactions personnelles. Ecocert, une entreprise qui a commencé en France en 1991, inspecte environ 70\% de l'industrie bio en France et environ 30\% dans le monde. La plupart des producteurs auxquels nous avons parlé semblaient accueillir à bras ouvert le concept de certification. L'éleveur ovin Dédou a lui-même participé à Ecocert, évaluant des producteurs dans d'autres pays européens et d'autres régions françaises, et même des producteurs à bien plus petite échelle, comme la boulangère Anne-Marie, considéraient la certification comme une évidence, défendant l'idée que la certification valait bien le coût pour la valeur ajoutée et le respect qu'elle donne au produit, spécifiquement en terme de gain de nouveaux clients. La certification donne encore plus de possibilités pour le travail libre. De nombreux fermiers bio de longue date commencent à embaucher des stagiaires de lycées agricoles publics, et passent aussi des accords d'apprentissage plus informels. Micias voyait la certification de ses sirops comme une nécessité et, grâce aux subventions conséquentes de l'État, les revenus de la culture biologique étaient plus élevés que le coût de la certification. Ces subventions sont entrain d'être supprimées et bien que l'aide de l'Union Européenne soit maintenue, certains étaient sceptiques que l'État français (comparé à celui d'Espagne, par exemple) soit réellement enclin à promouvoir l'agriculture bio. 
L'étiquetage et la certification bio ont été critiqués dans le contexte américain car, en tant que processus mercantiles, ils ne s'opposent pas à l'économie capitaliste classique. Au contraire, la certification agit comme un obstacle à l'investissement, elle crée des gammes de prix plus élevées qui peut être capitalisées dans de plus hautes valeurs locatives, elle augmente les prix pour le consommateur sans vraiment bénéficier au producteur, et elle accroit la concurrence entre les fermiers et mène à la croissance de nouveaux marchés (Guthman 2004, 176). De la même manière que la production à grande échelle de produits bio menace les plus petits producteurs, dans la distribution aussi, les géants du bio altèrent le paysage. La coopérative Floréal de Limoux et son antenne d'Espéraza, Amaranthe, ont été créées (respectivement en 1981 et 2010) dans le but de soutenir les plus petits producteurs, que leur nourriture bio soit certifiée ou pas. Mais à cause de son appartenance au distributeur national Biocoop, Floréal doit uniquement avoir des produits certifiés bio dans ses rayons, la rendant inaccessible à des micro-producteurs non-certifiés. Les plus petites coopératives, par opposition, ne reçoivent pas les ristournes que Biocoop fait à de plus grands acheteurs. Alistair Smith, un des fondateurs de Floréal, estime que la certification est avant tout un mécanisme mercantile, ce qui veut dire qu'elle est à la merci des efforts des lobbyistes pour rendre les standards moins rigoureux. Il trouve qu'Ecocert et Biocoop favorisent l'agrobusiness au final. Quelques-uns des plus petits producteurs que nous avons interrogés ne participent pas à Ecocert et disent que les formes dominantes de certification privilégient le 'grand bio' par rapport aux gens comme eux.

Mais une agriculture écologiquement durable et socialement juste qui gagne la confiance des consommateurs peut réussir sans aide de l'État ou de certifications délivrées par le marché. Certains dans la Haute Vallée ont choisi de travailler avec des processus de certification donnés par l'ONG bio-régionale Nature et Progrès-Aude, qui est très active dans la région. Grâce à un accord avec Biocoop, Floréal est en capacité de vendre des produits certifiés par Nature et Progrès. Bien qu'étant la première organisation à avoir établi le standard bio en France, Nature et Progrès s'est trouvée de plus en plus marginalisée dans les discussions concernant les certifications parce que son processus est plus rigoureux.

La vrai matérialisme ne met pas le profit au centre des échanges, le remplaçant par le processus de production, la qualité du produit et les relations entre producteur, marchandises et clients. La confiance occupe une place importante dans la pérennisation de la clientèle, mais un tel lien est vulnérable, son pouvoir restant limité. Le capitalisme se sert de la certification pour s'approprier les bienfaits du bio, offrant à ses consommateurs sa valeur et son capital de confiance qui s'étend de manière rhizomatique à toute la société.

Beaucoup d'alternatifs produisent et vendent des biens alimentaires agricoles, les marchés de petits producteurs et la confiance dans la nourriture bio localement produite résident donc au centre de leur économie alternative (voir aussi le cas d'Aide au Maintien d'une Agriculture Paysanne (AMAP), Blanc 2012). En addition aux critiques de la certification bio, il y a aussi un grand nombre d'études cruciales sur les réseaux d'alimentation alternative et sur leur hypothèse que la production et la consommation localisée de nourriture sont, en elles-mêmes, un développement positif pour le système alimentaire mondial. La défense du 'local' échoue à mettre en perspective les relations inégales entre les différentes localités, tout comme les hiérarchies sociales de race, de genre et de classe qui sont localement spécifiques mais à échelle mondiale (Dupuis et Goodman 2005; Hinrichs et Kremer 2002; Slocum 2006). Contrairement à de nombreux autres réseaux américains d'alimentation alternative, José Bové (2001) et François Dufour ont adopté une position complexe au sujet de la nourriture, du lieu de production et du commerce mondial n'ayant recours ni à la nostalgie pour le passé, ni au nationalisme (Massey 2005, 170). De même, nous ne voyons pas un recours au localisme défensif dans l'économie alternative de l'Aude. De ce que nos interlocuteurs nous ont dit, l'Aude n'avait pas de produits ou de méthodes exceptionnelles définis par le territoire, peut-être à cause de la pauvreté de la région. Ce que les petits producteurs ont introduit n'était pas circonscrit localement par une culture et une histoire spécifique et profondément enracinée. ${ }^{12}$

\footnotetext{
${ }^{12}$ Pour une analyse des perspectives chercheurs anglo vs. franco-méditerranéens du 'local', de l'utilité de la notion 'terroir' et des Systèmes Agroalimentaire Localisés, voir Bowen et Mutersbaugh (2014).
} 


\section{Echapper au travail aliéné}

Sur la route principale entre Carcassonne et les Pyrénées vers le sud se trouve un énorme chapeau melon posé sur un pylône, un des nombreux souvenirs de l'identité d'une ville de la Haute Vallée, Espéraza, un ancien centre de production français de chapeaux en feutre. Dans les années '30 la ville se targuait de 14 usines employant plus de 3,000 travailleurs, mais les chapeaux de feutre sont devenus passés de mode vers la moitié du $20^{\text {ème }}$ siècle, et la population a décliné de manière constante depuis la fin des années '60s, ne comptant que 2,100 habitants aujourd'hui.

Comme les autres villes de la Haute Vallée, Espéraza est un endroit difficile pour monter une entreprise. Le déclin de la population et la pauvreté se sont aggravés avec la concurrence du supermarché Intermarché voisin, ses panneaux publicitaires hurlant ses prix avantageux, coiffés du slogan "Tous unis contre la vie chère!" Les rues centrales de la ville sont dominées par des espaces commerciaux vides, les devantures en bois obsolètes de magasins vides depuis des décennies sont entrecoupées de victimes plus récentes. Le dimanche, malgré tout, Espéraza est transformée, son marché vivace drainant des clients de toute la Haute Vallée.

Les marchands arrivent tôt pour installer leurs produits, et dès $8 \mathrm{~h}$ du matin quelques-uns des petits producteurs bio sont assis à une des tables du café central, discutant et se relaxant. Au fil des années ils sont devenus des amis proches, connaissant les familles de chacun et leurs problèmes financiers, s'entre-aidant de manières variées.

Un de ces producteurs est Anne-Marie Verdonque, une boulangère née dans une famille de la classe ouvrière à Lille, une femme dont l'histoire démontre le sens aigu de dévotion que beaucoup ont ressenti par rapport à la campagne et la vie alternative. Mince et pleine de grâce, avec une force musculaire évidente, 'Marie' a accueilli Teresa chez elle dans un hameau reculé, portant une chemise tie-dye caractéristique de la Haute Vallée avec des pantalons noués par un cordon à la taille. Autour d'une tisane, elle a décrit sa vie précédente et sa décision de déménager à la campagne.

Ma vie était urbaine dans tous les sens du terme.... Urbaine en tout cas, urbaine mais on s'était quand même rapprochés de la nature et de la mer. Mais moi j'ai passé 20 ans à Lille donc je ne savais pas faire pousser un légume, je savais rien de la campagne.... Au départ je voulais vivre à la mer, près d'un élément. Mais après quand t'es citadine, tu sais pas ce qu'il faut faire à la montagne. J'avais peur des insectes, des souris, c'était un monde hostile pour moi. A la mer il n'y a pas trop de bêtes encore.

Ni Marie ni son compagnon n'ont trouvé de travail à Dunkerque, alors ils sont rentrés à l'intérieur des terres à St. Quentin, près de ses parents. A ce moment-là, Marie a ressenti une attirance profonde pour la fabrication de pains. "Maintenant je peux comprendre le symbolisme, mais à l'époque où j'avais envie de faire du pain, je ne savais pas pourquoi je voulais faire du pain." A St. Quentin elle a rencontré une âme sœur:

Lui il faisait ça car c'était une passion, il n'avait pas spécialement besoin d'argent, mais il faisait du pain pour quelques clients qu'il avait, et donc deux fois par semaines j'allais faire du pain avec lui. Et donc le pain le plus traditionnel qui soit, il n'y a pas besoin de grand chose pour faire son levain, il faut juste de la farine et de l'eau. Donc on prépare son levain, ça dure trois jours, et ensuite on prélève avant chaque pétrie un bout de levain et c'est toujours la même chose. C'est très très très simple. On ne peut plus simple. Le produit en lui même contient tout ce qu'il faut pour faire un bon pain. Il suffit d'ajouter de l'eau, du sel dans une bonne farine et du levain pour faire un bon pain. Et donc je suis un peu tombée dedans, cette histoire de pain ça m'a vraiment plu.

Comme d'autres boulangers que nous avons interrogés, Marie n'aimait pas seulement la simplicité mais aussi l'universalité du processus de fabrication: "On utilise les quatre éléments, il y a la terre, l'eau, l'air, le feu." Elle préférait travailler seule, complètement immergée dans le flux du processus. 
Marie et son partenaire de l'époque voulaient déménager dans le sud. Initialement, ils voulaient aller dans les Landes, mais une rencontre fortuite lui a montré que la Haute Vallée correspondait plus à ce qu'elle recherchait. "Et puis on sentait que dans ce pays, contrairement aux Landes où c'est des grandes exploitations avec beaucoup de maïs, on gave les oies, on fait du foie-gras, on fait de l'argent quoi... On exploite... Ici on trouvait plus un mode de vie à échelle humaine..." A la recherche d'un terrain, ils se sont liés immédiatement avec un couple vivant déjà dans le hameau Les Sauzils, qui les a accueillis avec enthousiasme et a aidé Marie à s'installer. Alors que le prix bas du terrain était un facteur majeur dans la décision de Marie de déménager dans la Haute Vallée, encore plus prépondérant était l'accueil chaleureux des alternatifs qu'elle a rencontrés, et plus généralement le sens de communauté venant de gens qui partagent la même cause. Elle ne recherchait pas seulement la nature et un endroit pour travailler, mais une communauté qui inspirerait et soutiendrait la vie qu'elle voulait mener.

\section{Au-delà du métro-boulot-dodo}

Comme Suzanne Berger le raconte, la génération européenne de 1968 n'a pas seulement rejeté la domination et l'inégalité illégitimes, mais aussi l'industrialisation de l'abondance elle-même:

...ce qui semble avoir été en jeu était une explosion de doute au sujet de la qualité et de la direction de la vie dans les sociétés industrielles avancées, au sujet du genre de relations humaines développées par les sociétés de consommation de masse, de l'incompatibilité des genres d'organisations requises pour diriger une société industrielle avec les valeurs et les relations personnelles qui sont nécessaires pour la satisfaction de besoins profondément humains. C'était une protestation non pas contre l'incapacité de l'État et de la société à fournir une croissance économique et une prospérité matérielle, mais contre leur succès bien trop considérable à avoir réussi cela, et contre le prix de ce succès (Berger 1979, 32).

Comme plusieurs milliers de personnes comme eux qui ont migré des villes vers les zones rurales éloignées à partir des années '60, presque tous nos participants nous ont dit avoir été à la recherche d'une vie qui échappe à la routine du métro-boulot-dodo et de l'hyperconsommation de l'Europe industrialisée. Mireille, l'apicultrice hollandaise, avait vagabondé dans le sud à la recherche de quelque chose de différent:

Je travaillais à la bibliothèque en Hollande et je voulais faire quelque chose avec la nature et je n'y arrivais pas parce que beaucoup de choses avaient à voir avec l'argent, le luxe et la société de consommation, alors j'ai pris mon vélo, j'ai tout vendu en Hollande et j'ai commencé à faire du vélo... Je ne savais pas vraiment ce que je voulais mais je ne voulais pas rester là. Quand j'étais à Carcassonne, je devais décider si j'allais continuer en Espagne ou si j'allais devoir travailler un peu. C'était en juin et il y avait Philippe assis sur une terrasse, c'est un bel homme et il m'a demandé si je voulais voir ses abeilles... et j'ai dit oui, donc... nous nous sommes mis ensemble! Il travaillait avec un grand apiculteur, ils travaillaient sur des projets pour Royal Jelly et il n'aimait pas ça du tout parce que c'est très strict. Tu travailles dans un laboratoire, c'est... [seulement] un peu de temps à l'extérieur. Je suis resté avec lui et doucement nous nous sommes mis ensemble et après deux ans de Royal Jelly nous avons décidé de créer notre propre entreprise de miel.

Beaucoup des petits producteurs étaient avides d'exprimer que leur sens de la vocation avait été soutenu par le bien-être et le plaisir du processus de travail lui-même. Travailler à l'air libre dans leur ferme au sommet de la colline rend l'agriculture agréable, disaient Dédou et Mireille. Isabelle voyait une continuité entre les impératifs écologiques qui sous-tendent son travail, la santé de sa famille et son plaisir au travail. Ayant étudié l'agronomie, elle comprit non seulement que l'agriculture industrielle allait transformer l'Europe en 'poubelle', mais que les pesticides sont dangereux pour la santé des familles de fermiers et, au travers d'effets génétiques, pour la santé de leurs enfants et de leurs petits-enfants. "...Quel est le plaisir de faire de l'agriculture avec des gants, des boites, des lunettes, c'est fou quoi!" 


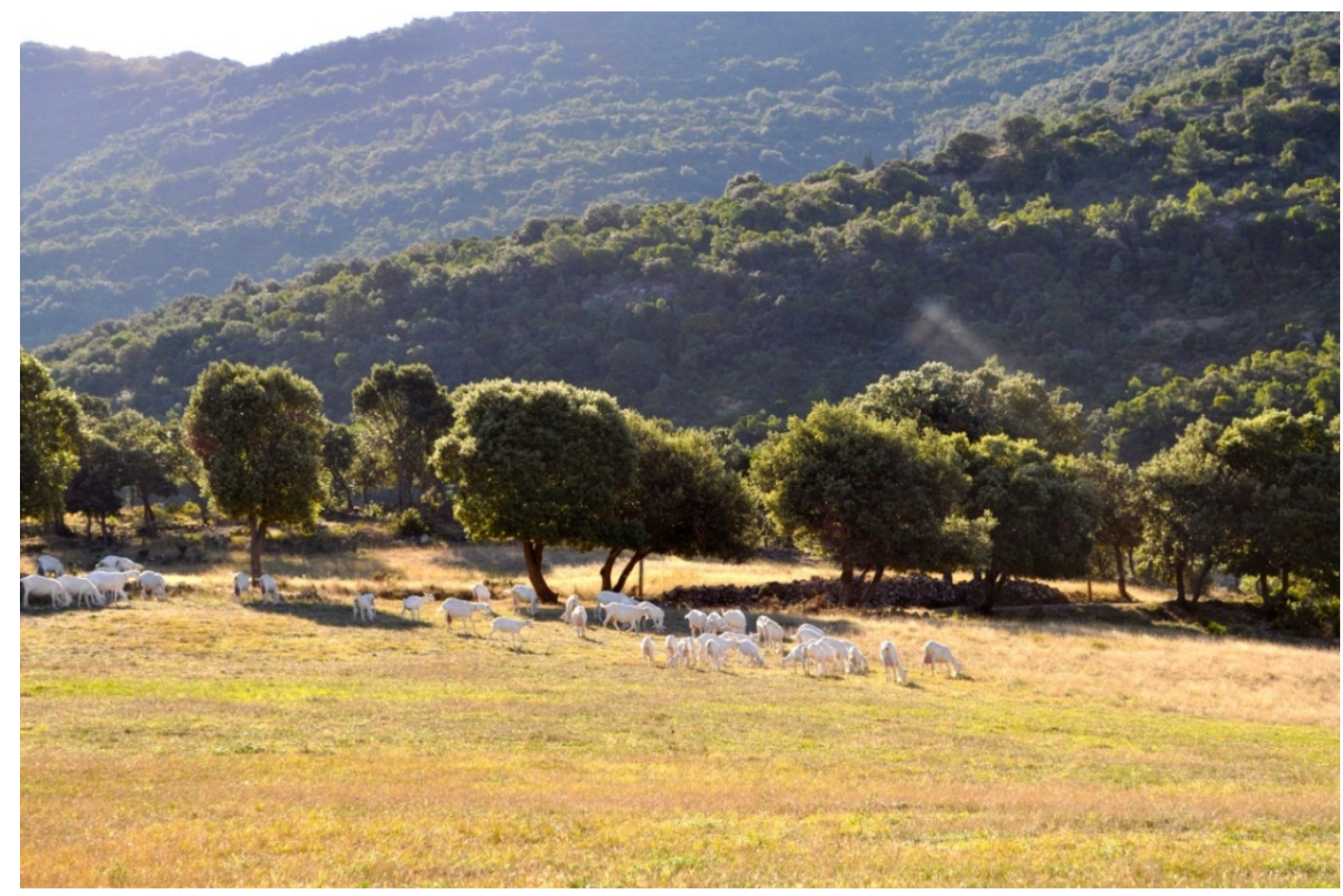

Figure 8: Les chèvres et garrigue chez Jean-Baptiste Gaschard, producteur de fromage de chèvre frais, Corbières. Photo prise par Rachel Slocum.

Beaucoup d'alternatifs sont très conscients que leur richesse ne se trouve pas seulement dans l'accès à une nature 'intacte', mais aussi parce qu'ils disposent de beaucoup plus de temps libre en comparaison avec la plupart des Français aux moyens modestes. Jean-Baptiste est fier de travailler moins d'heures que ses parents, par exemple. Ils sont conscients de la croissante taylorisation de l'espace domestique, de la frénésie interminable au travail et de la marchandisation intense et généralisée du temps (Figure 8). Ils ont souvent évoqué le rythme fou de la vie dans les grandes villes, et ils résistaient à faire des 'affaires', même quand ils subissaient eux-mêmes une pression considérable. L'esthétique dominante de l'interaction sociale est assez détendue et sympathique, et il est considéré comme impoli et aussi malsain de courir ça et là en parlant au téléphone.

Le musicien Patrick Terris est l'un des plus déterminés à combattre la tyrannie des affaires (Figure 9). Parmi les plus anciens vendeurs au marché d'Espéraza, son petit stand présente son pain d'épices et ses gâteaux, tout comme de la moutarde et un jus fait par ses parents octogénaires et son ancienne partenaire Claude, tous d'anciens membres de la commune de Canterate. L'entreprise de Patrick est petite et ses revenus sont très modestes, mais il apprécie profondément son mode de vie, pour lequel il a abandonné Paris quand il avait la vingtaine. Il peut travailler dans la beauté et la tranquillité de Canterate, il voit ses nombreux amis au marché et il a assez de temps pour jouer de multiples instruments dans des groupes différents. Même après 35 ans, la réponse habituelle de Patrick quand on lui demande comment il va, est de répondre qu'il est difficile d'être malheureux quand on a la chance d'être accueilli tous les jours par les pics enneigés de Saint Barthélémy.

D'autres producteurs ont amené une éthique de travail plus rythmée à leur existence artisanale ou alternative, se lançant dans des projets qui requièrent beaucoup de travail intensif. Néanmoins, même pour ces travailleurs durs à la tâche, la politique anticonsumériste et les critiques du travail formel ordinaire restent très fortes. La dynamique Isabelle refuse des requêtes de magasins qui veulent vendre ses produits parce que 
"nous on n'a pas très envie de faire des kilomètres pour donner des légumes, on fait deux marchés et on vend tout. Il faut être beaucoup plus grand...et on ne veut pas."

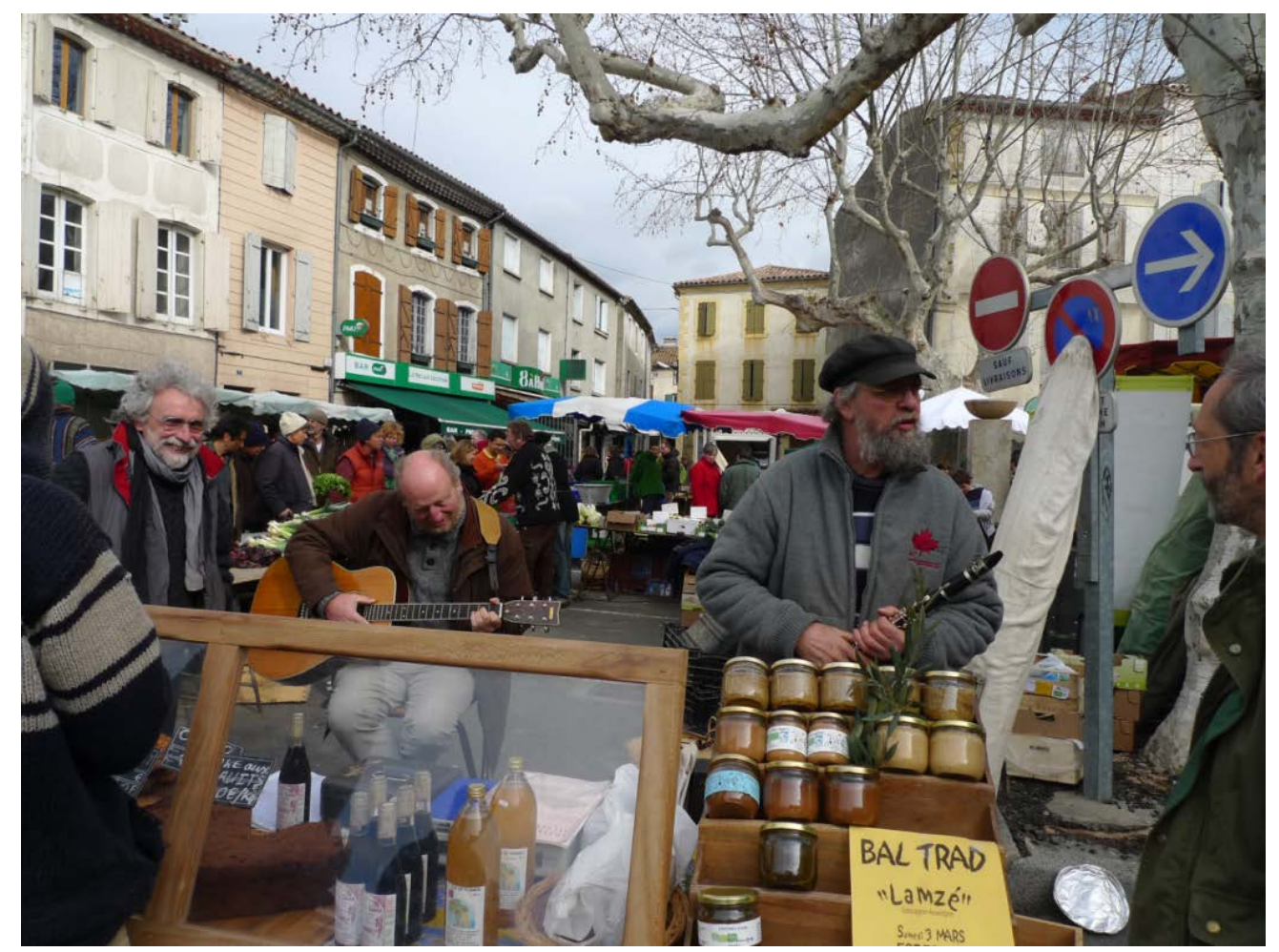

Figure 9: Le stand de Patrick Terris, marché Espéraza, Haute Vallée. Photo prise par Teresa Gowan.

Ce que tous les alternatifs semblent apprécier dans leur vie quotidienne n'est pas uniquement le sens donné à leur travail, mais aussi le degré d'autonomie extraordinaire accordé au comment et dans une certaine mesure, au quand ils travaillent. Le travail est bon, même apprécié et recherché, mais l'état d'occupation forcé demandé par un job (avec une grimace) est grandement déploré. Le boulanger Rolf a exprimé très clairement le lien entre la vrai matérialisme et la libération du travail aliéné. Selon lui, le caractère de son riche pain noir est l'expression directe de son autonomie:

Moi, je fais peu de pain, je vends tout mon pain mais j'exige que les gens le prennent comme il est. Je ne fais pas trente variétés. Je ne demande pas aux gens ce qu'ils veulent, tu vois, je ne me laisse pas tirailler par la demande—c'est moi qui propose mais comme je le fais au mieux je trouve aussi des personnes qui le veulent. J'essaye de trouver un équilibre, et pour ça c'est très important que je travaille ma peur. Si j'avais peur de ne pas pouvoir vivre de ce que je fais, alors je ne pourrais pas faire des produits de qualité, c'est comme ça que je le vois.

\section{Une nouvelle répartition du temps}

Si la vrai matérialisme donne aux produits artisanaux ou faits à la maison une valeur qui dépasse l'aspect financier, le travail significatif et plaisant, le gain de temps et l'autonomie substantielle dans le processus de production artisanal sont également importants pour protéger la vie du producteur du réductionnisme imposé par la relation d'échange purement financière. Le rejet par le producteur du métro- 
boulot-dodo casse la division marquée du marché entre 'l'exploitation-acceptable-car-génératrice-d'argent' et le 'loisir-en-tant-que-dépense-d'argent.' Il n'y a désormais plus rien à gagner à réduire le temps passé à la maison dans le but de soutirer un peu de trésorerie de tout travail, et ce pour acheter les marchandises de loisirs qui ont remplacé la qualité de vie.

\section{Résister à l'aliénation à la nature}

Chacun des principes de la plénitude peut être lu comme une réponse aux quatre types d'aliénation que Marx décrit dans ses Manuscrits de 1844 (Marx 2007: 67-80). Au lieu du producteur aliéné du produit de son propre travail, la vrai matérialisme engage le travailleur dans une relation durable avec sa création, une relation qui peut s'étendre en fin de compte jusqu'au consommateur. De manière similaire, le besoin d'accomplissement, de plaisir, et d'autonomie des producteurs repousse clairement le second typel'aliénation du processus de travail.

La libération dont Rolf, Marie, Mireille ou Patrick ont fait l'expérience est dans une certaine mesure un produit du privilège blanc. Qu'importe leur pauvreté, ils ont tous migré vers l'Aude pour suivre un rêve, plutôt que pour échapper aux aspirations entravées ou aux difficultés dans les anciennes colonies.

Par exemple, Sami, cultivateur de légumes né en Algérie, nous a clairement dit qu'il avait été forcé de faire du jardinage.

Sami: Je suis venu en '88 parce qu'il y avait des problèmes en Algérie et tout ... et j'ai commencé à travailler [dans la culture maraîchère] avec [mon père]. Parce qu'il n'y a pas autant de travail, c'est difficile...

Rachel: Vous avez cherché à faire autre chose?

Sami: Oui, mais parce que, moi, à la base en Algérie, j'étais enseignant quand même. [De] littérature.

Rachel: Ah oui, c'est difficile de laisser tomber ça.

Sami: Eh oui... Je n'avais pas le choix.

Au cours des années Sami avait essayé de faire du mieux que possible dans les circonstances qui étaient les siennes: "Alors maintenant je suis là, ici, ça me plaît, la nature, le contact avec les gens comme ça, j'avoue que c'est très important. Si je ne le fais plus un jour, ça va me manquer. Ah oui." Sami ne pouvait pas voir son travail comme une sorte d'évasion absolue de l'exploitation.

Il a néanmoins apprécié le contact direct avec la nature. La troisième considération de Marx est que la privatisation de la nature par le capitalisme nous sépare de notre 'être générique', de notre besoin humain profond d'interagir directement avec le monde naturel en tant que "matériel, objet et instrument" de notre créativité (Marx 2007, 74).

En s'installant dans l'Aude rurale, les migrants n'y ont pas seulement vu la beauté visuelle intacte de la garrigue rocheuse des Corbières ou les falaises spectaculaires et les forêts de la Haute Vallée-ils ont vu la possibilité de prendre part à la nature, de travailler avec le terrain et les animaux d'une manière collaborative et durable (voir aussi de Sartre, Douence, et Mercier 2010). Premièrement, cela voulait dire vivre très simplement. Beaucoup ont commencé en louant ou en achetant du terrain conjointement sans permis de construire, où ils ont commencé par installer des caravanes, des yourtes ou d'autres logements basiques. Neuf d'entres-eux (Isabel, Beatrice, Rolf, Patrick, Claude, Brigitte et Gerard, et Dominic et Myriam) avaient commencé leur vie dans la région comme membres de communautés se réappropriant lentement des hameaux ruinés ou abandonnés. Dans leur commune à Canterate, par exemple, Claude et Patrick ont attendu l'eau courante pendant 18 ans. Il y a de nombreuses années le maçon, fromager et musicien Sylvain Dumas a d'abord vécu dans une maison sans électricité et eau courante. Lorsque nous lui avons demandé pourquoi il avait choisi de vivre de cette façon, Sylvain nous a dit, "c'était l'époque ...la fin des années 1970, 1968, on disait qu'il était 'interdit d'interdire'...Oui, la façon dont je vivais était marginale mais plus tranquille." Aujourd'hui Philippe et Mireille vivent avec bonheur dans une maison avec deux chambres, une pompe 
manuelle pour l'eau et pas de douche, de l'électricité ou d'un téléphone portable. Dominic et Myriam, et plus tard leur fils Micias, ont progressivement construit de simples maisons de bois autour de vieux vans délabrés

La façon la plus directe de résister à la domination du capitalisme industriel et à l'abondance sans âme était de créer un mode de vie moins dépendant des combustibles fossiles, pas seulement au travers de la production bio, mais plus généralement en tirant le nécessaire de la vie quotidienne plus directement de la nature, sous la forme de légumes du jardin, de fruits, d'herbes médicinales, de champignons forestiers et de bois de chauffage. Glaner directement de la nature reste central au mode de vie alternatif et la cueillette, le jardinage, la mise en conserve et d'autres activités en tout genre faites à la maison sont toujours très répandues, tout comme la création d'objets artistiques et esthétiques et d'espaces construits avec des objets récoltés de la nature. Viser l'autosuffisance totale est néanmoins souvent vu comme à la fois impossible et tributaire d'un isolement non-nécessaire.

\section{L'approvisionnement communal et la nature sociale des liens économiques}

La quatrième forme d'aliénation selon Marx est "l'aliénation de l'homme à l'homme" (2007:77)—elle souligne la violence immense faite à la subjectivité humaine alors que la déshumanisation et les conditions de production qui relèvent de l'exploitation disparaissent derrière le voile d'une relation financière délocalisée.

En général les producteurs prenaient grand soin d'éviter l'exploitation des autres, eux qui s'étaient battus si vaillamment pour conquérir leur autonomie. Quelques producteurs plus établis prenaient des stagiaires. La plupart du temps ce sont des arrangements informels mais les stagiaires viennent des programmes d'agronomie des lycées environnants. Tout aussi importants sont les WWOOFers ${ }^{13}$ (des bénévoles qui échangent du travail manuel dans des fermes durables pour une chambre et de la nourriture, créant des liens avec la vie de fermier et parfois apprenant les compétences pour commencer leur propre petite entreprise). Alors que le stage peut être une pratique de travail problématique, nos interlocuteurs semblaient prendre très sérieusement le devoir de former les autres à devenir la prochaine génération de producteurs, d'aucuns parlant d'anciens stagiaires qui ont réussi dans toute l'Europe (et même le monde).

Ce qui était plus important économiquement dans toutes les communautés alternatives était la relation forte entre l'approvisionnement informel et le communautarisme, ou au moins la création de liens sociaux forts. Le désir passionné d'autosuffisance domestique et communautaire (l'autarcie) s'est dissipé au profit d'un approvisionnement communautaire dans la production et la consommation locale (Cazella 2001). Une grande partie de cette production prend néanmoins place en dehors de l'échange mercantile.

La génération '68 était motivée par un sens fort de la vie communautaire comme forme idéale d'arrangement social. Initialement les soixante-huitards organisaient la vie communautaire et leur travail autour d'une vision du monde radicale partagée. Alors que deux de nos participants étaient en désaccord, la plupart d'entres-eux pensaient que les projets formels avaient connu moins de succès que les communautés construites autour d'un communautarisme informel ${ }^{14}$ où ils travaillaient ensemble de manière plus incrémentale et avec de moins grandes ambitions (voir aussi Gibson-Graham 2003).

De tels arrangements illustrent les bases économiques de la communauté-impliquant qu'une économie alternative peut être fondée, en fait, sur une communauté alternative, et vice versa. En particulier il vaut la peine d'explorer en détail la question du troc, de l'échange informel en dehors de l'économie monétaire, une forme primaire qui permet l'indépendance collective plutôt que l'indépendance privée ou personnelle.

\section{Les réseaux de troc}

Dans la Haute Vallée plusieurs participants ont mentionné à quel point le coût de la vie était inhabituellement 'bas.' Il est vrai que les loyers tendent à être moins chers que la moyenne et certainement bien plus bas que dans les grandes villes, mais ni l'eau, le gaz, ou l'électricité ni les produits du supermarché

\footnotetext{
${ }^{13}$ Le mot est derivé de l'acronyme de 'World Wide Opportunities on Organic Farms.'

${ }^{14}$ Le sens dans lequel nous comprenons la centralité du don et de la coopération dans l'économie alternative en tant que 'communitariste' diffère donc en caractère du moralisme américain d'Amitai Etzioni (1994) ou Robert Bellah (1992) tout comme d'autres formes de collectivisme plus fermement organisés.
} 
ne sont plus bas que dans le reste de la France. La vie devient de plus en plus abordable pour ceux qui font de l'auto-approvisionnement et pratiquent des loisirs sans frais dans la nature, mais une autre raison pour laquelle la vie est plus abordable est l'ubiquité de l'échange informel, qui permet même aux membres les plus modestes des réseaux sociaux alternatifs de bénéficier de travail occasionnel ou de production au niveau domestique sans être empêtré dans la législation volumineuse française.

Des produits de producteurs échangés, des outils, des revenus dans certains cas et d'autres échanges de travail conséquents. Dans un arrangement qui a duré plusieurs années, Rolf a fourni Max avec son riche pain noir, en échange de tous les légumes dont il avait besoin. Patrick échange l'utilisation et l'entretien permanent d'un tracteur contre de l'aide pour s'occuper de ses vaches.

L'économie du troc s'étend loin au-delà des producteurs du marché eux-mêmes. Une femme à Lagrasse pratique l'obstétrique en échange de nourriture, une autre s'occupe des comptes pour une petite entreprise et reçoit des soins vétérinaires pour ses animaux. Dans le Val de Dagne de nombreux parents ont échangé au cours des années du labourage, de la construction, des légumes, du jardinage, du pain ou de la menuiserie contre des leçons de musique de Tessa, la mère de Teresa. Dans tous ces cas, la relation fonctionne car il n'y a pas de comptabilité. Un tel comptage saperait l'aspect du don.

\section{Le danger de l'exclusion}

La centralité des réseaux informels soulève néanmoins des questions au sujet du coût de la vie pour les personnes qui restent en dehors du cercle pour différentes raisons (voir aussi la conclusion de Deverre et Lamine 2010). La population immigrée du département, en particulier, a peu de chances d'intégrer les réseaux artisans-alternatifs. Alors que pour de nombreux Français blancs natifs issus de la classe ouvrière, les revenus conséquents de l'économie dominante sont un but plus séduisant, le travail agricole rappelant trop le faible statut social et le travail difficile d'un passé paysan largement rejeté. En général, les migrants d'Afrique du Nord qui ont vécu dans la région depuis deux ou trois générations sont concentrés dans les grandes villes et ne participent pas à l'économie alternative, bien qu'ils jouent un rôle conséquent dans la revente d'une grande variété de produits sur les marchés locaux (voir Tchoukaleyska 2009).

Nous n'avons pas trouvé de preuves d'exclusion active; certains des producteurs offraient un échange de travail avec un Franco-Algérien (Kasim) et un groupe de jeunes Polonais qui sont arrivés dans la Haute Vallée au début des années 2000. Cependant, ces jeunes gens avaient déjà constitué un 'capital' alternatif en termes de connaissance, ou même de compétences. Tout simplement avoir un sentiment assez profond de ce qui est en jeu et établir des connexions assez fortes pour faire partie de cette économie peut être suffisant pour beaucoup de monde. Par exemple, Sami, plus vieux, s'est trouvé isolé du réseau d'alimentation alternatif des Corbières bien qu'il puisse bénéficier d'autres économies morales dans sa communauté de Carcassonne. Avec un racisme systématique qui bouche l'accès à tant d'opportunités aux groupes non-blancs des anciennes colonies (Stoler 2005), ajouté à la grande crise de l'embauche qui fait face à la jeunesse française en général, la question de l'exclusion doit être posée.

\section{Coup de main}

Nous avons trouvé plus de récits que prévu au sujet d'échanges informels entre les anciens Audois et les nouveaux arrivants, nous poussant à spéculer que la force du troc était due à une synthèse des pratiques paysannes et des principes non-conformistes des alternatifs. Assise dans son salon ensoleillé débordant de signes de sa vie trépidante d'arboricultrice et d'organisatrice de la communauté, Claude expliquait comment la communauté qui avait rendu vie au hameau abandonné de Canterate avait organisé l'échange d'une façon très schématique, seulement pour pouvoir apprendre des plus vieux campagnards du coin comment aborder l'échange d'une façon plus générale et informelle:

Avant le SEL on avait un cahier de troc, c'est-à-dire qu'on avait un cahier sur chaque double page, on avait une personne avec qui on troquait. Sur la page de gauche c'est ce que moi je prends a Monsieur X, et sur la page de droite ce que Monsieur X me prend...Pour chaque personne avec qui on avait des échanges on note tout sur ce cahier...c'était en argent, 
équivalent en argent. On était très content avec ça. Ça marchait bien. D'ailleurs c'est assez typique de la campagne, je pense à cette idée de troc, moi, je connaissais pas, moi je n'avais jamais pratiqué le troc avant, mais on a eu ça dès le début, quand on est arrivé ici en 1980, ce sont nos voisins qui étaient de vieux paysans, très très âgés qui sont venus nous voir pour nous proposer de faire de la pomme de terre ensemble, parce ce que nous on avait plein de main d'œuvre, on était très nombreux a l'époque, eux ils n'étaient pas nombreux mais eux ils avaient un tracteur, eh donc, l'idée c'était de faire ensemble les pomme de terre. Ils nous ont achetés nos plants, eux ils ont acheté leurs plants, nous on a nos terres, eux ils ont leur terre, mais on cultive ensemble chez nous et ensemble chez eux. C'était superbe, c'était génial.

Le dynamisme de Claude a poussé sa vision de l'échange sans cash vers de plus grands projets. "La chose la plus extraordinaire à laquelle j'ai pris part, en tant que membre de Canterate, était le système d'échange local, le SEL. Donc vous êtes intéressés dans la manière dont nous survivons par ici? Et bien le SEL a été incroyablement important pour ça... Donc le SEL est un système de troc multilatéral—l'idée vient du Royaume-Uni, vous savez? Et le premier SEL en France a été créé..." Claude fit une pause pour l'effet, "ici! A Canterate!"

Le SEL a attiré 500 membres au travers de l'Ariège et de l'Aude, et les organisateurs ont rapidement étendu le procédé à d'autres régions de France, avec même François Terris qui a fait une apparition à la télévision nationale. Les membres pouvaient faire don d'un bien ou d'un service à une autre personne et recevoir d'une autre. Les gens passaient des annonces dans le journal, proposant des services ou des biens, que ce soit du jardinage, des gâteaux au chocolat, des pommes, des transports en voiture réguliers vers Toulouse ou des massages. L'organisation (qui n'avait pas d'employés) organisait aussi des marchés populaires où les marchandises étaient échangées avec le crédit de grains de sel (le nom de la monnaie alternative du système) de chaque membre.

Claude était la présidente pendant les quatre premières années et la secrétaire pour quatre années de plus, mais elle y a perdu beaucoup d'énergie et l'organisation a connu un déclin. Cependant, certaines personnes de la Haute Vallée sont toujours impliquées dans le mouvement SEL et, plus généralement, l'enthousiasme des première années à donné un grand coup de fouet à la prise de conscience des possibilités du troc, laissant derrière eux toutes sortes de relations qui fonctionnaient toujours bien. Par exemple, Claude a décrit comment elle avait vraiment besoin d'aide avec la récolte de cassis après le départ d'un de ses partenaires de troc. Elle a utilisé le système SEL pour débaucher des gens pour l'aider avec la récolte et les a payés avec de la confiture ou du sirop de cassis. Au fil des années ils sont devenus amis et ils utilisent toujours le même arrangement pour les récoltes. La plupart des récoltes des petits producteurs sont facilitées par le troc, tout comme de nombreux projets de construction.

Le SEL n'a pas seulement servi à développer l'économie d'échange local, il a aussi créé une quantité de nouvelles connexions, particulièrement avec les arrivées récentes. Parlant de l'analyse des systèmes d'échange local, le sociologue français Smaïn Laacher $(2002,82)$ souligne que "trop souvent les questions au sujet de leur pertinence ont été de nature économique: le rôle économique de la devise, la valeur monétaire des biens et des services d'échanges, etc." Mais ces systèmes permettent aussi la création de liens sociaux très concrets, en fournissant un espace pour "l'expérimentation avec de nouvelles formes de relations sociales à l'intérieur d'une économie non-monétaire" (Laacher 2002:83).

\section{Le rôle de l'État social dans l'approvisionnement collectif}

Parmi nos participants, le rejet radical des liens avec l'État était peu commun. Les alternatifs condamnaient régulièrement le rôle des gouvernements français et européens dans la promotion des intérêts des supermarchés, des banques, des compagnies d'énergie et d'autre formes de capital, produisant de la surconsommation à la maison, la guerre ainsi que des inégalités démesurées et mondiales à l'étranger. Cependant, nombre de ces alternatifs étaient beaucoup moins critiqués à l'égard des fonctions sociales et réglementaires de l'État.

"En France, si tu n'as pas d'argent, déjà au niveau médical ce n'est pas mal, parce que là en France tu as tout gratuit. Quand on n'a rien, c'est, plutôt au niveau de la santé, eh, c'est plutôt bien", dit Rolf "[T]u te 
trouves aussi un logement. Le minimum est toujours donné par l'État." Pour les producteurs français, et dans une certaine mesure les immigrants d'autres pays de l'Union Européenne comme Rolf, ça relevait du bon sens que l'État social soit nécessaire et dans bien des cas essentiel à leur survie-fournissant des retraites, des allocations aux familles avec des enfants, subventionnant le logement, l'énergie et la nourriture pour les sansemplois, et octroyant la couverture maladie universelle et couvrant les frais de scolarité dans l'enseignement secondaire pour la population entière. En effet, nous nous sommes demandées si cette relation était devenu si banale qu'elle en était sous-évaluée-les gens considéraient seulement rarement que ces sources de soutien puissent être fondamentalement mises en danger par les méfaits du casino capitaliste ou les tournants libéraux à l'intérieur du gouvernement français.

Habituées aux paysages plus inégaux et précaires des États-Unis, nous avons été frappées par les pressions financières relativement légères subies par de nombreux parents, qui avaient plus tendance à mentionner des difficultés à prévoir des déplacements au cinéma ou acheter des vêtements de marque. Les enfants des petits producteurs vivaient simplement, mais leurs activités ne coûtaient pas grand-chose à leurs parents. Comme d'autres enfants de la campagne, ils prenaient les transports publics pour aller à l'école ou au sport, et les parents pouvaient se reposer sur la haute qualité et le coût bas de l'assurance maladie. Myriam haussa les épaules:

Non, les enfants ne représentaient pas vraiment beaucoup de dépenses du tout jusqu'à ce qu'ils soient au lycée où nous avons dû faire face au problème des grandes marques. Et c'est à ce moment qu'avoir à les conduire partout est plus devenu un problème. Mais c'était quelques années vraiment. C'est surtout après 10 ans, à onze ans quand ils rentrent au collège, où là, il $\mathrm{y}$ a une demande des enfants au sujet de la consommation, avant non, ouf, nous, on a jamais connu ça, maintenant peut être que c'est plus difficile....

Avec la crise économique ralentissant les ventes, de nombreux nouveaux petits producteurs avaient eu des difficultés à faire des ventes et ils étaient devenus plus dépendants d'une aide de l'État initiée en 2009, le Revenu de Solidarité Active, qui augmente le revenu de travailleurs indépendants vivant sous le seuil de pauvreté et aide les sans-emplois. A l'intérieur des réseaux plus alternatifs, au moins, il ne semblait pas y avoir de honte à faire la demande du RSA, du moment que vous montrez que vous faites quelque chose de votre vie. "C'est vraiment pour les gens qui travaillent. Il y a un petit groupe de petits producteurs comme nous par ici qui reçoivent le Revenu de Solidarité Active", nous a dit un producteur, soulignant 'l'active.' Ce producteur semble avoir voulu se différencier de l'aide qui datait d'avant 2009, le Revenu Minimum d'Insertion, avec ses connotations de 'passivité.' "Ça fait vivre le coin", dit-il sérieusement. "C'est vraiment pour les gens qui travaillent. Lorsque tu proposes un travail de 300 Euros (US \$336), ils te donnent des Euros supplémentaires pour vivre. Il y a plusieurs personnes dans le coin qui reçoivent ce revenu solidaire actif. C'est comme les agriculteurs - on a de l'aide parce qu'on a des activités. On est assez nombreux, en effet. Ça fait vivre le coin." Alors que de nombreuses personnes dans le coin ont leurs problèmes financiers, le filet de sauvetage en dessous d'eux est substantiel comparé à ceux d'autres pays.

Pendant ce temps-là certains des plus alternatifs continuent à s'en sortir presque totalement en dehors de l'économie formelle au travers d'un mélange d'auto-approvisionnement et de troc, échangeant du travail informel contre de la nourriture, un endroit où rester ou d'autres biens. C'était le cas de Kasim, un constructeur écolo et autodidacte autoproclamé, que nous avons rencontré alors qu'il échangeait du travail avec un des cultivateurs de légumes bio les plus prospères de la Haute Vallée, Max. Nous avons parlé avec Kasim à cette époque-là, il travaillait dans l'impressionnante ferme écologique de Max, renforçant ses murs avec du chanvre résistant à l'extérieur et de la terre locale à l'intérieur. Avec le troc Kasim a eu le droit de vivre sur le terrain de Max dans une caravane avec un vue sur les contreforts des Pyrénées à droite et son œuvre à gauche, se nourrissant avec les produits de Max, lisant et partageant du temps entre amis pendant son temps libre.

Mince et athlétique, avec un visage enjoué et expressif, Kasim expliquait que le travail de fermier ne l'inspirait pas en lui-même. "J'ai besoin d'être créatif!" Il nous expliqua ce qui l'avait amené vers la construction écologique. "J'aime bien revenir vers quelque chose, le regarder et me dire 'je l'ai fait!"' Alors qu'il nous montrait les mécanismes d'un barrage et un système d'irrigation sur le terrain, un ancien projet pour 
Max, Kasim expliqua qu'il s'était enseigné lui-même des pratiques écologiques de toute l'Europe, aimant les défis présentés par la construction alternative. Offrant du travail de construction en troc, Kasim est capable de gagner assez d'argent pour vivre chaque été dans l'Aude, s'en sortir et même voyager pendant les mois d'hiver. Des artisans très à l'écart de la marchandisation comme Kasim semblent servir d'ancrage au caractère alternatif de l'Aude, pas seulement comme supports de travail vitaux pour les petits producteurs mais comme icones de libération de la routine économique capitaliste.

\section{Au-delà de la relation financière délocalisée}

Marx a développé l'idée que c'est l'échange financier qui médiatise la valeur dans une économie capitaliste, oblitérant les relations politiques, sociales, environnementales et culturelles actives dans la production, et réduisant à la fois les biens et leurs concepteurs à une valeur d'échange hautement contingente mais apparemment inhérente. Toute transaction faite au travers de l'échange de marchandises devient automatiquement définie par ces échanges.

Comme nous l'avons vu, les petits producteurs de l'Aude, en tandem avec les plus grandes communautés qu'ils servent, ont développé une multitude de stratégies pratiques pour résister à l'effet nivelant de l'argent. Leur 'vrai matérialisme' se manifeste dans une profonde identification avec le produit tout au long de son existence, avec la supposition que le travail de fabrication et de vente devrait être un travail gratifiant, autonome et non aliéné. La vente elle-même est relocalisée-un processus de vente directe qui illumine et n'obscurcit pas le processus de production.

La vision alternative du monde est renforcée par les courants forts du discours antimondialiste français (Waters 2010) dans les médias régionaux et nationaux, renforçant généralement les idéaux de la décroissance, la rétrogradation et l'anticonsumérisme (Latouche 2006). Un bihebdomadaire éponyme (La Décroissance) est devenu un important centre de communication et de discussion, touchant avec son ton sans prétention les membres les plus jeunes et souvent les moins bien éduqués de la communauté depuis plus d'une décennie. Bien plus qu'aux États-Unis, une discussion au sujet des économies alternatives a touché de nombreux publics à travers la France, avec une discussion dirigée par le Parti pour la décroissance, la Confédération paysanne, l'Institut des études sociales et économiques pour une décroissance soutenable et de nombreux autres intellectuels publics.

\section{Les dangers de la dette et de l'argent}

Nos participants semblaient habitués à ces débats - certains de manière très subjective, d'autres très informés. Peut-être sans surprise, le sujet sur lequel les gens se sont exprimés avec le plus de vigueur était le besoin de prendre de la distance par rapport à la domination de l'argent. La plupart, comme Marie, reniaient explicitement la valeur supposée suprême de l'argent:

Teresa: Et ça s'est vraiment la chose qui est distincte ici, il y a plusieurs gens qui pensent comme ça, l'argent c'est pas la première chose.

Marie: Et non. On fonctionne dans un monde d'argent donc il faut aussi avoir les pieds sur terre, il faut gagner de l'argent, car on nous demande de payer les assurances, les trucs, les machins, il faut aussi gagner de l'argent. Je cherche pas à m'en foutre plein les poches. Je m'en fous.

Il y avait une crainte particulière pour le danger de la dette. Dédou nous disait avec force que le conseil le plus important qu'il pouvait donner aux nouveaux arrivants est de commencer très petit. Les prêts de la banque, nous expliqua-t-il, contraignent les fermiers à rester dans l'agriculture et le marketing dominant à la fois directement (dans les stipulations des prêts) et indirectement (en créant le besoin d'une rentrée d'argent substantielle immédiate).

Mireille et Philippe ont commencé avec un extracteur vieux de 80 ans et un camion qui devait être poussé pour démarrer, persuadés qu'ils ne s'endetteraient pas. La plupart des apiculteurs utilisent un bras mécanique pour porter les charges lourdes impliquées dans l'apiculture mais Philippe et Mireille ont refusé 
d'emprunter les 14,000 Euros (US \$15,680) qu'il coûtait. En fait, ils n'utilisent pas des stagiaires parce que ça ressemblerait trop à de l'exploitation de demander à des travailleurs non-rémunérés de soulever de telles charges:

Nous avions la même idée, nous ne voulions pas emprunter d'argent du tout parce que nous étions tous les deux trop effrayés par la dette. Avoir les grosses machines ou les gros investissements, ça changerait nos vies. Alors nous avons décidé de tout faire à la main... Quand nous sommes allés au marché, nous avions vu des gens malheureux parce qu'ils avaient pris le risque d'investir beaucoup d'argent [dans leur entreprise]. Nous nous sommes dits, pas question. Nous ne voulons jamais rentrer dans ce système. On a toujours eu la chance qu'on était bien ensemble et que quand on était au marché on a vu tous les gens qui avait investi beaucoup d'argent et qui étaient très malheureux à cause de ça. On se dit jamais on va rentrer dans ce système. Ça c'était clair.

\section{Vers une politique de la plénitude}

Qu'est-ce que cette étude sur les petits producteurs de l'Aude nous suggère au sujet d'un futur anticapitaliste, ou au minimum moins capitaliste? Au cours des récentes décennies, le bas coût de l'entrée dans la production à petite échelle est devenu plus difficile à atteindre. D'abord la région de Lagrasse et puis la Haute Vallée sont devenues de plus en plus populaires en termes de tourisme et chez les gens en recherche d'une maison de campagne, particulièrement les émigrés britanniques enthousiastes de trouver un marché de l'habitat français si abordable. Cette immigration a eu des effets inégaux sur nos participants, à la fois stimulant le marché des gites et celui de certains produits et entraînant une montée conséquente des prix des maisons qui a rendu la tâche plus difficile pour les petits producteurs de s'installer. Par-dessus l'augmentation des prix des propriétés, la crise a ajouté un fardeau supplémentaire. Alors que nous écoutions les producteurs, nous avons été frappées par les difficultés que certains d'entres-eux affrontaient.

Des Français plus pauvres ont néanmoins dû retourner vers les zones rurales au cours des 20 dernières années (Pagès 2005), attirés par le bas coût de la vie, et la crise actuelle semble avoir accéléré cette tendance à la fois dans les Corbières et la Haute Vallée, avec de nombreux jeunes, à la fois Français et internationaux, se lançant dans la micro-production dans des circonstances très marginales. Réussiront-ils? Est-il toujours possible pour des jeunes gens de commencer avec littéralement rien d'autre qu'une tente et un sac-à-dos? Il semble clair que ceux qui vivent très modestement sans se sentir 'pauvres' peuvent le faire plus facilement que dans la plupart des endroits du monde entier grâce à des allocations de chômage et des allocations familiales décentes, les pensions et les retraites, et une assurance maladie à bas prix. Les lois comme le fermage, le RSA et l'État social français en général ont été pour nous des découvertes étonnantes; nous sommes plus habituées à la destruction persistante de l'état social aux États-Unis. Cette relation contiguë entre les indemnités attribuées par l'État et l'expérimentation économique créative suggère qu'une 'politique de la plénitude' ferait bien de considérer la sécurité sociale elle-même comme une forme d'approvisionnement collectif et que même une petite aide du gouvernement peut-être d'une importance économique vitale.

Les pratiques économiques diverses des alternatifs de l'Aude sont reliées entre-elles par l'approvisionnement communautaire. Ce cas affine notre compréhension d'anciens débats autour de la nécessité et de l'attrait de l'autosuffisance. L'expérience audoise suggère que le don peut être une alternative fondamentale à la pérennité des économies alternatives, tout en soulevant des questions au sujet du coût de la vie pour ceux qui sont exclus de la participation à de tels réseaux d'échange. Une politique de la nouvelle richesse dans une société démocratique qui aspire à l'égalité des chances ne peut pas mettre de côté le problème de l'exclusion systémique. Si le but est d'étendre les économies qui échappent à la routine capitaliste, alors une politique de la plénitude devra trouver les moyens de créer des formes d'abondances différentes ou des conceptions de l'abondance attractives pour plus de gens que ceux qui ont un goût pour la contre-culture. Dans les zones rurales, ça doit peut-être vouloir dire participer au développement agricole 
durable et au travail de justice globale de la Confédération paysanne, si alternatif et paysan sont des positions subjectives qui peuvent coexister dans la Confédération. ${ }^{15}$

Aux défaitistes qui voient le néolibéralisme derrière toutes les portes, nous trouvons beaucoup de choses enthousiasmantes dans les économies alternatives de l'Aude avec leurs pratiques bien développées et articulées de la nouvelle matérialité et du travail non aliéné. Comme Schor et Laacher nous voyons une forte symbiose entre la prise de distance par rapport au capitalisme routinier et la restauration de réseaux de connexions humaines plus riches. La centralité de l'approvisionnement collectif au travers du troc, du coup de main, de la non-concurrence et des coopératives tenues par des bénévoles encourage les gens à comprendre la survie individuelle comme faisant partie d'une entreprise collective, renforçant donc la communauté avec des relations particulièrement réciproques et collectives plutôt qu'orientée principalement vers la consommation individualisée. A l'intérieur d'une culture politique anticonsumériste, la restriction intentionnelle des besoins et des désirs à l'intérieur de l'économie monétaire se convertit en un indéniable surplus de temps et de travail investis dans les projets de chacun—le surplus domestique ou individuel est finalement transformé en un bien collectif ou au moins en circulation.

L'échelle de notre analyse - une plongée dans les vies des producteurs individuels—nous rappelle que la domination du capitalisme routinier prend racine dans la vie quotidienne des individus, tout comme dans les grandes institutions qui dominent le système mondial. La décision explicite de participer à un mode de vie alternatif semble avoir donné aux petits producteurs de la Haute Vallée et des Corbières un sens particulièrement fort de bien-être et de confiance en leur survie, soulignant le caractère politico-culturel inhérent des arrangements économiques. L'accès au terrain lui-même, avec ses plaisirs gratuits manifestes et ses opportunités d'auto-approvisionnement, la culture étendue du troc et du coup de main, et, à des degrés variables, le soutien de l'État français autorisent les petits producteurs à éloigner la vie quotidienne de l'anxiété créée par le consumérisme et d'autres déformations de l'expérience humaine imposées par les arrangements économiques capitalistes. Poursuivre la plénitude n'est pas seulement une stratégie économique mais aussi une politique et une esthétique, comme l'apicultrice Murielle l'a expliqué de manière succincte:

C'est la base du capitalisme, c'est la consommation. C'est la consommation. Mais ça c'est toujours un choix de vie après. Les gens s'ils veulent ils peuvent faire quelque chose encore. Ils sont encore assez libres de faire ca. Il faut surtout que tu luttes. Moi je trouve qu'il faut lutter contre ce système capitaliste. Je pense trouver la créativité dans la vie sans consommer. C'est la créativité qui amène le bonheur, ce n'est pas l'argent.

\section{References}

Bebbington, A.J. and Batterbury, S.P.J. 2001. Transnational livelihoods and landscapes: political ecologies of globalization. Cultural Geographies 8(4): 369-464.

Bellah, R. N. 1992. The good society. New York: Vintage.

Berger, S. 1979. Politics and antipolitics in Western Europe in the seventies. Daedalus 108(1): 27-50.

Birchfield, V. 2005. José Bové and the globalisation countermovement in France and beyond: a Polanyian interpretation. Review of International Studies 31(3): 581-598.

Blanc, J. 2012. Construire l'alternative agro-alimentaire: ressorts sociaux et politiques du déploiement des AMAP en Île-de-France. Norois 224:21-34.

Bové, J. 2001. The world is not for sale: farmers against junk food. London: Verso.

Bowen, S., and T. Mutersbaugh. 2014. Local or localized? Exploring the contributions of FrancoMediterranean agrifood theory to alternative food research. Agriculture and Human Values 31(2): 201213.

Buller, H. 2003. De la terre au territoire: the reinvention of French rural space. Modern and Contemporary France 11 (3): 323-334.

${ }^{15}$ José Bové, après tout, n'a pas eu un patrimoine paysan (Birchfield 2005). 
Burke, B.J. and B.W. Shear. 2014. Introduction: engaged scholarship for non-capitalist political ecologies. Journal of Political Ecology 21: 127-221.

Cazella, A.A. 2001. Les installations agricoles nouvelles: le cas des agriculteurs néo-ruraux dans l'Aude (France). Espace, populations, sociétés 1(2):101-108.

Collard, R-C., J. Dempsey, and J. Sundberg. 2015. A manifesto for abundant futures. Annals of the Association of American Geographers 105(2):322-330

Cortes-Vazquez, J.A. 2014. A natural life: neo-rurals and the power of everyday practices in protected areas. Journal of Political Ecology 21: 493-515.

de Sartre, X.A., H. Douence and C.-E. Mercier. 2010. Choisir et redéfinir le local l'exemple d'un type de filières courtes: les AMAP en Béarn. In J. B. Traversac and S. Villard (eds) Les circuits courts alimentaires Paris: Educagri. Pp 100-120.

Deffontaines, J.-P. 2005. Le terroir, une notion polysémique. In L. Bérard et al. (eds) Biodiversité et savoirs naturalistes locaux en France. Montpellier: CIRAD, IDDRI, IFB, INRA. Pp. 38-43.

Demossier, M. 2003. Rural France in Europe: new challenges. Modern and Contemporary France 11(3): 265278.

Deutsch, T. 2010. Building a housewife's paradise: gender, politics, and the emergence of supermarkets, 1919-1968. Chapel Hill: University of North Carolina Press.

Deverre C. et C. Lamine. 2010. Les systèmes agroalimentaires alternatifs: une revue de travaux anglophones en sciences sociales. Economie Rurale 317: 57-73.

Dupuis, E.M. and D. Goodman. 2005. Should we go 'home' to eat?: toward a reflexive politics of localism. Journal of Rural Studies 21(3): 359-371.

Escobar, A. 1996. Constructing nature: elements for a poststructural political ecology. In R. Peet and M.J. Watts (eds). Liberation ecologies: environment, development, social movements Routledge: London. Pp. 46-68.

Etzioni, A. 1994. The spirit of community: the reinvention of American society. New York: Touchstone.

Fantasia, R. 1995. Fast food in France. Theory and Society 24(2): 201-243.

Fickey, A. 2011. 'The focus has to be on helping people make a living': exploring diverse economies and alternative economic spaces. Geography Compass 5(5): 237-248.

Gibson-Graham, J.K. 1996. The end of capitalism (as we knew it): a feminist critique of political economy. Cambridge, MA: Basil Blackwell.

Gibson-Graham, J.K.. 2003. Enabling ethical economies: cooperativism and class. Critical Sociology 29(2): 123-161.

Gibson-Graham, J.K.. 2006. Diverse economies: collaboration and community in economic geography, Annual Progress in Human Geography lecture. Paper read at Annual Meeting of the Association of American Geographers, Chicago, IL.

Gilg, A.W. and M. Battershill. 1999. The role of household factors in direct selling of farm produce in France. Tijdschrift voor Economische en Sociale Geografie 90(3): 312-319.

Glassman, J. 2003. Rethinking overdetermination, structural power and social change: a critique of GibsonGraham, Resnick and Wolff. Antipode 35(4): 678-698.

Gowan, T. and R. Slocum. 2014. Artisanal production, communal provisioning and anti-capitalist critique in the Aude, France. In J. Schor and C.J. Thompson (eds.) Sustainable lifestyles and the quest for plenitude: case studies of the new economy. New Haven: Yale University Press. Pp.27-62.

Grasseni, C. 2004. Skilled landscapes: mapping practices of locality. Environment and Planning D: Society and Space 22(5): 699-717.

Grasseni, C. 2009. Developing skill, developing vision: practices of locality at the foot of the Alps. New York: Berghahn Books.

Grasseni, C. 2013. Beyond alternative food networks: Italy's solidarity purchase groups. London: Bloomsbury. 
Grasseni, C. 2014. Seeds of trust. Italy's gruppi di acquisto solidale (solidarity purchase groups). Journal of Political Ecology 21: 179-192.

Guthman, J. 2004. Agrarian dreams: the paradox of organic farming in California. Berkeley, CA: University of California Press.

Haraway, D. 2014. SF: string figures, multispecies muddles, staying with the trouble. Talk at the University of Alberta, March 24, 2014. [accessed March 1, 2015]

Hervieu, B., and F.B. Purseigle. 2008. Troubled pastures, troubled pictures: French agriculture and contemporary rural sociology. Rural Sociology 73(4): 660-683.

Hinrichs, C. and X. Kremer. 2002. Social inclusion in a Midwest local food system project. Journal of Poverty 6 (1): 65-90.

Hinrichs, C.C. 2000. Embeddedness and local food systems: notes on two types of direct agricultural markets. Journal of Rural Studies 16: 295-303.

Holloway, L. and M. Kneafsey. 2000. Reading the space of the farmers' market: a preliminary investigation from the UK. Sociologia Ruralis 40(3): 285-299.

Hughes, A. 2005. Geographies of exchange and circulation: alternative trading spaces. Progress in Human Geography 29(4): 496-504.

Jarosz, L. 2011. Nourishing women: toward a feminist political ecology of community supported agriculture. Gender, Place and Culture 18(11): 307-326.

Laacher, S. 2002. Les systèmes d'échange local (SEL): entre utopie politique et réalisme économique. Mouvements 1: 81-87.

Latouche, S. 2006. Le pari de la décroissance. Paris: Fayard.

Lawson, V. 2005. Hopeful geographies: imagining ethical alternatives. Singapore Journal of Tropical Geography 26(1): 36-38.

Leyshon, A. 2005. Introduction: diverse economies. Antipode 37(5): 856-862.

Lyon, S. 2014. Fair trade towns USA: growing the market within a diverse economy. Journal of Political Ecology 21: 146-160.

Marx, K. 2007. Economic and philosophic manuscripts of 1844. Mineola, NY: Dover Publications.

Massey, D. 2000. Entanglements of power: reflections. In J.P Sharp, P. Routledge, C. Philo and R. Paddison (eds.) Entanglements of power: geographies of domination/resistance. London: Routledge. Pp. 279286.

Massey, D. 2005. For space. London: Sage.

McCarthy, J. 2006. Neoliberalism and the politics of alternatives: community forestry in British Columbia and the United States. Annals of the Association of American Geographers 96(1): 84-104.

Meadows, D.H., D.L. Meadows, J. Randers, and W.W. Behrens III. 1972. The limits to growth: a report for the Club of Rome's project on the predicament of mankind. New York: Universe Books.

Oberhauser, A. M. 2005. Scaling gender and diverse economies: perspectives from Appalachia and South Africa. Antipode 37(5): 863-874.

Ozer, P., and M. Bay. 2009. Alimentation durable: de la promotion des filières courtes à la promotion d'emplois durables. Liège: Université de Liège.

Pagès, A. 2005. La pauvreté en milieu rural. Toulouse: Presses Universitaires du Mirail.

Portes, A., M. Castells, and L. Benton. 1995. The informal economy: studies in advanced and less developed countries. Baltimore, MD: Johns Hopkins University Press.

Robbins, P. and K. Monroe Bishop. 2008. There and back again: epiphany, disillusionment, and rediscovery in political ecology. Geoforum 39(2): 747-755.

Saldanha, A. 2006. Re-ontologizing race: the machinic geography of phenotype. Environment and Planning D Society and Space 24 (1): 9-24.

Saldanha, A. 2011. Race. In J. Agnew and J. Duncan (eds) Companion to human geography. London: Sage. Pp 453-464. 
Schor, J. 2010. Plenitude: the new economics of true wealth. London: Penguin Press.

Schor, J. and C.J. Thompson eds. 2014. Sustainable lifestyles and the quest for plenitude: case studies of the new economy. New Haven: Yale University Press.

Slocum, R. 2006. Anti-racist practice and the work of community food organizations. Antipode 38(2): 327349.

St. Martin, K. 2005. Mapping economic diversity in the first world: the case of fisheries. Environment and Planning A 37: 959-979.

Stoler, A.L. 2005. Racist visions for the 21st century: on the banal force of the French radical right. In J.-A. Lee and J. S. Lutz (eds.) Situating 'race' and racisms in time, space, and theory: critical essays for activists and scholars. Montréal: McGill-Queen's University Press. Pp.114-137.

Tchoukaleyska, R. 2009. The markets of Montpellier: food, culture, identity and belonging in France. MA thesis, Vancouver, British Columbia, University of British Columbia.

Thompson, C.J. and G. Coskuner-Balli. 2007. Enchanting ethical consumerism. Journal of Consumer Culture 7(3): 275-303.

Tsing, A. 2009. Supply chains and the human condition. Rethinking Marxism 21(2): 148-176.

Tsing, A. L. 2012. Empire's salvage heart: why diversity matters in the global political economy. Focaal (64): 36-50.

Waters, S. 2010. Globalization, the confédération paysanne and symbolic power. French Politics, Culture and Society 28(2): 96-117.

Zakaria, F. 2011. The post-American world: release 2.0. New York: W.W. Norton. 